\title{
Flora en playas y dunas costeras de México
}

\section{Flora on beaches and coastal sand dunes of Mexico}

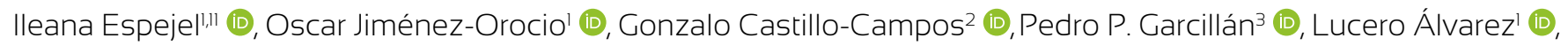

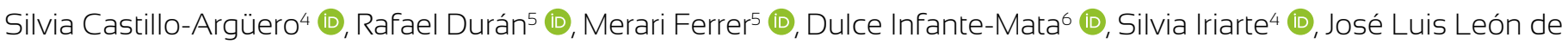

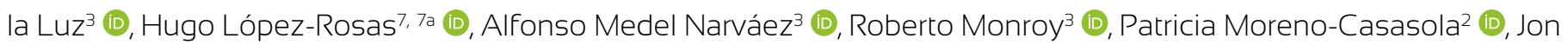 \\ P. Rebman (iD, Natalia Rodríguez-Revelo' (D), Jesús Sánchez-Escalante ${ }^{9}$ (D), Sula Vanderplankº (i)
}

1 Universidad Autónoma de Baja California, Facultad de Ciencias, Apdo. postal 1880, Ensenada, B. C., México.

2 Instituto de Ecología, A.C., Carretera antigua a Coatepec 351, El Haya, 91070 Xalapa, Veracruz, México.

3 Centro de Investigaciones Biológicas del Noroeste, Avenida Instituto Politécnico Nacional 195, Playa Palo de Santa Rita Sur, 23096 La Paz, B.C.S., México.

4 Universidad Nacional Autónoma de México, Facultad de Ciencias, Avenida 3000, Circuito Exterior s/n, Delegación Coyoacán, Ciudad Universitaria, O4510 Cd. Mx., México.

5 Centro de Investigaciones de Yucatán, calle 43 No. 130, Colonia Chuburná de Hidalgo, 97200 Mérida, Yucatán, México.

6 El Colegio de la Frontera Sur, Departamento de Manejo Sustentable de Cuencas y Zonas Costeras, Carretera antiguo aeropuerto km 2.5, 30700 Tapachula, Chiapas, México.

7 Universidad Nacional Autónoma de México, Instituto de Ciencias del Mar y Limnología, Estación El Carmen, México.

7aDirección actual: Centro del Cambio Global y la Sustentabilidad en el Sureste. Calle Centenario del Instituto Juárez $\mathrm{s} / \mathrm{n}$, Colonia Reforma, 86080 Villahermosa, Tabasco, México.

8 San Diego Natural History Museum, 1788 El Prado, 92101 San Diego, CA, Estados Unidos de América.

9 Universidad de Sonora, Luis Donaldo Colosio s/n, entre Sahuaripa y Reforma, colonia centro, 83000 Hermosillo, Sonora, México.

1OBotanical Research Institute of Texas, 1700 University Drive, 76107 Fort Worth, TX, Estados Unidos de América.

11 Autor para la correspondencia: ileana.espejel@uabc.edu.mx

Citar como:

Espejel, I., O. Jiménez-Orocio, G. Castillo-Campos, P. P. Garcillán, L. Álvarez, S. Castillo-Argüero, R. Durán, M. Ferrer, D. Infante-Mata, S. Iriarte, J. L. León de la Luz, H. López-Rosas, A. Medel Narváez, R. Monroy, P. Moreno-Casasola, J. P. Rebman, N. Rodríguez-Revelo, J. Sánchez-Escalante y S. Vanderplank. 2017. Flora en playas y dunas costeras de México. Acta Botanica Mexicana 121: 39-81. DOl: http://dx.doi. org/10.21829/abml21.2017.1290

Recibido: 4 de enero de 2016. Revisado: 19 de enero de 2017. Aceptado: 26 de mayo de 2017

DOI

http://dx.doi.org/10.21829/abml21.2017.1290

\section{Resumen:}

Antecedentes y Objetivos: Se presenta el primer listado florístico de las playas y dunas costeras de México, actualizado y respaldado por ejemplares de herbario.

Métodos: Se revisaron 14 herbarios nacionales y extranjeros. Se sobrepuso una retícula de $2 \times 2 \mathrm{~km}$ al mapa de la costa y de las dunas costeras de México para referir todos los registros que corresponden a 2180 sitios con 12,419 ejemplares de plantas. Se calcularon índices de diversidad, similitud, diversidad taxonómica y redundancia.

Resultados clave: La flora registrada consta de 153 familias, 897 géneros y 2075 especies de plantas vasculares, las cuales representan 9.5\% de la flora vascular de México. Las cinco colecciones con índices de redundancia altos IR $\geq 0.7$ son los herbarios de la Universidad Nacional Autónoma de México (MEXU, IR=0.9), del Centro de Investigación Científicas de Yucatán (CICY, IR=0.8), y los del Centro de Investigaciones Biológicas del Noroeste (HCIB), el Instituto de Ecología, A.C. (XAL) y el del Museo de Historia Natural de San Diego (SD), cada uno con un IR de 0.7. Se reconocen cinco regiones florísticas que corresponden a los mares de México. El Pacífico Norte tiene mayor diversidad taxonómica y el Pacífico Sur menor diversidad taxonómica. El Golfo de California, Golfo de México y Mar Caribe tienen diversidades taxonómicas similares. Las especies con más registros son Trianthema portulacastrum (165), Croton punctatus (107), Echites umbelllatus (106) e Ipomoea pes-caprae (90). Por el carácter de ecotono de las dunas costeras, la mayoría de las especies se comparten con los tipos de vegetación vecinos (selvas, matorrales, y humedales como las marismas y manglares).

Conclusiones: Esta primera lista de la flora en playas y dunas de México es la base para múltiples estudios florísticos regionales y locales, biogeográficos, y ecológicos; además, que será importante para su uso en temas de impacto ambiental y manejo costero.

Palabras clave: análisis florísticos nacionales, ecosistemas costeros, ecotono, plantas vasculares, vegetación costera.

\section{ABSTRACT:}

Background and Aims: The first updated floristic checklist, supported with herbarium specimens, is presented for beaches and coastal sand dunes of Mexico.

Methods: The analysis is based on the revision of 14 national and foreign herbaria. A $2 \times 2 \mathrm{~km}$ grid was placed over a map of the coastal sand dunes of Mexico to reference the herbarium records, corresponding to the 2180 sites and 12,419 examples of plants documented. Indices of diversity, similarity, taxonomic diversity, and redundancy were calculated.

Key results: The flora of the beaches and coastal sand dunes of Mexico consists of 153 families, 897 genera, and 2075 species of vascular plants. These represent $9.5 \%$ of the vascular flora of Mexico. The herbaria with high indices of redundancy ( $I R \geq 0.7$ ) are the herbarium of the Universidad Nacional Autónoma de México (IR=0.9), the Centro de Investigación Científica de Yucatán (CICY, IR=0.8), the Centro de Investigaciones Biológicas del Noroeste (HCIB), the Instituto de Ecología, A.C. (XAL) and the Natural Museum in San Diego (SD), each with an IR of 0.7. Five floristic regions are clearly recognized, which correspond to the seas of Mexico. The North Pacific has the highest taxonomic diversity and the South Pacific has the least. The Gulf of California, Gulf of Mexico and the Caribbean Sea have very similar taxonomic diversity. The species with the most recorded occurrences are Trianthema portulacastrum (165), Croton punctatus (107), Echites umbellatus (106) and Ipomoea pes-caprae (90). Considering the ecotonal nature of the ecosystem, most dune plants are shared with neighboring vegetation (forests, scrubs, wetlands, such as marshes or mangrove swamps).

Conclusions: This first checklist of the flora of beaches and coastal dunes is the baseline for multiple future studies: floristic, biogeographic, and ecological. Moreover, this list will be important if used in environmental assessments and coastal zone plans.

Key words: coastal ecosystems, coastal vegetation, ecotone, national floristic analyses, vascular plants. 


\section{INTRODUCCIÓN}

Los ecosistemas arenosos costeros constan básicamente de geoformas conocidas como dunas costeras, médanos, arenales o depósitos eólicos de arena, ligados a las playas (Goldsmith, 1989). Las playas y dunas son estructuras geomorfológicas con o sin vegetación que han motivado la investigación botánica y de ecología vegetal, porque son un ejemplo perfecto para ilustrar adaptaciones de las plantas al gradiente del ambiente marino-terrestre (Gallego-Fernández y Martínez, 2011). En las playas y dunas es posible estudiar los factores que gobiernan los sistemas de dunas y playas (viento, erosión/acresión, enterramiento, abrasión, inundación, sequía, aspersión salina y nutrientes escasos), ya que se expresan en gradientes espaciales muy marcados que los hace sistemas muy atractivos de estudio (Martínez y Moreno-Casasola, 1996; Martínez et al., 1997; Hesp et al., 2011). También resultan de interés las limitantes bióticas que dominan a las dunas estabilizadas por vegetación en suelos con mayor cantidad de nutrientes (p. ej., Jones et al., 2004; Lane et al., 2008), que generalmente se encuentran en el extremo del gradiente más distante del mar.

Aunque hay especies exclusivas de playas y dunas costeras, en estas últimas se forma una franja de ecotono con los tipos de vegetación que las flanquean (Castillo y Moreno-Casasola, 1996; Peinado et al., 2011), por lo que es frecuente encontrar elementos de matorral xerófilo (Vanderplank, 2011, observación personal de Natalia Rodríguez e Ileana Espejel), bosque de encino (Castillo et al., 2016), selva baja caducifolia (Castillo y TraviesoBello, 2006) y aún de humedales (Moreno-Casasola et al., 2009) en suelos arenosos. En Yucatán, Campeche, Chiapas y Oaxaca hay individuos de mangle botoncillo (Conocarpus erectus L.) en el primer cordón de dunas (Espejel et al., 2015). Castillo y Moreno-Casasola desde 1996 definieron un grupo de especies en dunas costeras compartidas con otros tipos de vegetación tierra adentro, formando parte de los ecosistemas mencionados. Por otro lado, Devall (2005) ejemplifica cómo especies que se consideran exclusivas de playas y dunas costeras (p.ej. Ipomoea pes-caprae (L.) R. Br.), cuando encuentran las condiciones adecuadas, se establecen en suelos arenosos como en el lago de Nicaragua. Por esta razón, es más preciso referirse a la vegetación en dunas costeras y no a la vegetación de dunas costeras.

Aunque se ha documentado que las dunas costeras prestan 23 servicios ambientales de los cuatro tipos (provisión, regulación, culturales y de soporte) (Everard et al., 2010), es posible que sean los sistemas con mayor presión antrópica en México (Martínez et al., 2014). Esto se debe a que las playas y dunas costeras son ecosistemas con alta importancia socioeconómica debido a las preferencias del turismo y la urbanización que éste genera, así como al desarrollo de la ganadería extensiva y al cultivo de cocotales (García-Mora et al., 2001; Hesp, 2004; Muñoz-Vallés et al., 2011). Por ejemplo, Jiménez-Orocio et al. (2014a) estiman que $1 \%$ de la superficie de México (aproximadamente 800,000 hectáreas) son dunas costeras y que $46 \%$ de ellas han sido transformadas (uso agropecuario o urbanizadas) (Jiménez-Orocio et al., 2014b). La mayoría de las dunas fuera de áreas naturales protegidas están muy deterioradas (fragmentadas, perforadas, invadidas de flora exótica) (Jiménez-Orocio et al., 2014c). Asimismo, al estar ubicadas en la zona más baja de las cuencas hidrológicas, son el vertedero de residuos generados cuenca arriba (basura, pesticidas, nutrientes, etc.), que no fueron completamente depurados en ecosistemas adyacentes como el manglar y otros humedales. En las playas también se encuentra basura proveniente del mar (Derraik, 2002).

Actualmente, las playas y dunas costeras aumentan su valor debido a los escenarios de cambio climático por elevación del nivel medio del mar (Cavazos et al., 2013), que favorecen al servicio ecosistémico de protección que las playas y dunas proveen a la infraestructura urbana y agropecuaria en zonas costeras (Mendoza-González et al., 2012).

En México, varios autores han estudiado la vegetación en playas y dunas costeras (p. ej., Johnson, 1977; 1982; Espejel, 1987; Castillo et al., 1991; Martínez et al., 1992; Castillo y Moreno-Casasola 1996; Peinado et al., 2011; Álvarez-Molina et al., 2012). Sin embargo, hay temas hiper-estudiados pero también enormes vacíos de 
conocimiento (Jiménez-Orocio et al., 2015). En especial, la información florística sobre playas y dunas de México estaba desactualizada (Moreno Casasola et al., 1998) y dispersa en fuentes bibliográficas o herbarios, por lo que era urgente contar con un inventario actualizado de la flora de sistemas arenosos costeros del país. La Comisión Nacional para el Conocimiento y uso de la Biodiversidad (CONABIO) financió una iniciativa para completar el conocimiento faltante (Espejel et al., 2015). Se planteó la siguiente pregunta: ¿cuál es diversidad florística en playas y dunas del país? Para complementar la respuesta también se preguntó: ¿quiénes son los principales colectores y donde están las colecciones regionales más completas de estos ecosistemas?, ¿qué sitios se han colectado mejor y donde es necesario colectar más?, y ¿cómo varían espacialmente las especies de plantas en un área geográfica tan grande y heterogénea como es el litoral mexicano? Por ello, el objetivo de este artículo es analizar la riqueza florística en playas y dunas costeras dentro de México con base en los registros de herbarios y colectas propias. El listado florístico, aunque por su propia naturaleza será siempre provisional, es de gran utilidad como elemento de referencia en estudios florísticos, ecológicos, biogeográficos, así como herramienta para el manejo integral de la zona costera mexicana.

\section{Materiales y Métodos}

El estudio fue realizado por un equipo de numerosos investigadores de varias instituciones del país: Universidad Autónoma de Baja California (UABC), Centro de Investigaciones Biológicas del Noroeste, S.C. (CIBNOR), Universidad de Sonora (USON), Instituto de Ecología, A.C. (INECOL), Centro de Investigación Científica de Yucatán, A.C. (CICY), Universidad Nacional Autónoma de México (UNAM) y de California, Estados Unidos de América, Museo de Historia Natural de San Diego (SDNHM) (Espejel et al., 2015). La información de cada grupo de investigadores se conjuntó en una única base de datos en MS Access, 2010.

Para comenzar se elaboró una lista provisional de plantas vasculares colectadas en playas y dunas costeras a partir de: a) base de datos de CONABIO con plantas registradas con las palabras clave playas, dunas costeras, médanos y arenales, b) especímenes en los 14 herbarios

Cuadro: Colecciones revisadas y colectas propias depositadas en 14 herbarios principales. En el Rancho Santa Anna (RSA), Desert Botanical Garden (DES) y University of California Berkeley (CAVA) hay respectivamente 5, 3 y 1 especies con sus respectivos ejemplares por lo que no fueron incorporados en el análisis estadístico. En XAL hay nuevos registros de G. Castillo que fueron agregados a este listado y en RSA están los registros de S.Vanderplank que no fueron agregados a este listado.

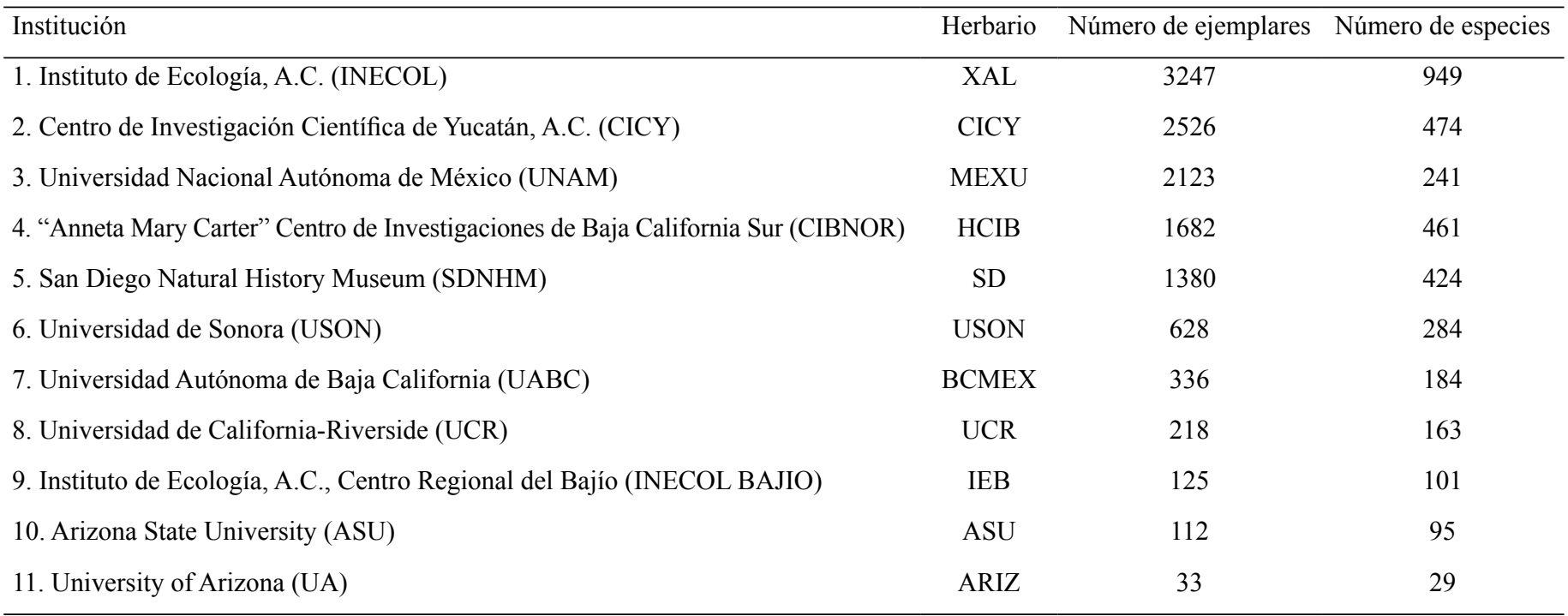


participantes (Cuadro) registrados con las mismas palabras clave que en a), y de colectores conocidos por sus colectas en playas y dunas costeras de México y c) registros bibliográficos (en especial Moreno Casasola et al., 1998).

Se revisó la base de datos resultante, se eliminaron registros bibliográficos no depositados en herbarios y registros georreferenciados incorrectamente. Para los registros de especímenes de herbario cuya localidad solo estaba descrita se calcularon las coordenadas geográficas consultando el marco geoestadístico del Instituto Nacional de Estadística y Geografía por municipios (INEGI, 2010). Para este artículo, se depuró la base de datos entregada a CONABIO, se agregaron especies que los autores colectaron ya finalizado el proyecto y se incorporaron otras especies que, por falta de datos, no fue posible incorporarlas al formato de CONABIO, en especial los ejemplares del Herbario Nacional de México (MEXU).

Se revisaron sinonimias en los catálogos recomendados por CONABIO: catálogo taxonómico de especies de México, en Capital Natural de México, vol. I: Conocimiento actual de la biodiversidad (CONABIO, 2009); Sistema Integrado de Información Taxonómica SIIT (CONABIO, 2015), y Tropicos.org (TROPICOS, 2016).

Para los análisis, se armó una cuadrícula sobre la franja de costa (Fig. 1), con celdas de $2 \times 2 \mathrm{~km}$. Se eligió este ancho porque corresponde al ancho promedio de las dunas nacionales, calculado a partir del mapa de dunas de Jiménez-Orocio et al. (2014a). Cada celda se tomó como una unidad de muestreo para los análisis de redundancia.

Se calculó un índice de redundancia para cuantificar el grado de "exhaustividad" (redundancia) de la colecta en cada una de las celdas de acuerdo a Garcillán et al. (2003).

$$
\mathrm{IR}=1-(\mathrm{S} / \mathrm{h})
$$

Donde,

$\mathrm{R}=$ redundancia,

$\mathrm{S}=$ número de especies y

$\mathrm{h}=$ número de ejemplares o registros.
Se calculó el IR para: 1) una región determinada, (2) colecciones y (3) colectores.

(1) La redundancia de una región se calculó utilizando las celdas, en este caso $2 \times 2 \mathrm{~km}$. Los valores de $\mathrm{S} \mathrm{y} \mathrm{h}$ corresponden a cada una de estas celdas, y se obtuvo información sobre la exhaustividad relativa de colecta entre las distintas celdas de la región.

(2) La redundancia de colecciones se hizo con el total de especies en la colección como S y el total de colectas en la misma como h.

(3) En la redundancia en colectores, el valor de S corresponde al total de especies encontradas por un colector dado, y h al total de especímenes colectados por dicho autor.

Para clasificar las regiones florísticas de playas y dunas costeras de México, se hizo un análisis de conglomerados con la presencia/ausencia de las especies, utilizando el índice de similitud de Pearson con el paquete estadístico Statistica 8 (StatSoft Inc., 2007), y se calculó el índice de Shannon para estimar la diversidad florística por región utilizando el paquete EstimateS (Cowell, 2013).

\section{Resultados}

La flora en playas y dunas de México está compuesta por 153 familias, 897 géneros y 2075 especies (Apéndice). Los autores, según su experiencia, reconocen que solo $4.6 \%$ de las especies (95) son preferentemente de playas y dunas costeras, las cuales corresponden básicamente a especies de playas y dunas semimóviles, ya que las dunas estabilizadas tienen una cobertura vegetal compartida con especies de los tipos de vegetación aledaños como son bosques o selvas tropicales, matorrales e inclusive especies de manglar y de humedales de agua dulce.

La variable independiente $\mathrm{x}$ es el número de especies/familia, y la dependiente es el número o porcentaje de familias. Al graficarla se obtiene la clásica "curva en forma de J" que se observa regularmente en estos casos (Fig. 2), ya que la gran mayoría de las familias (78\%) tiene menos de 10 especies. De hecho, casi la tercera parte (32\%) de las familias solo tiene una especie y únicamente cuatro familias (Asteraceae-248 especies, Fabaceae-223, Poaceae-168 y Euphorbiaceae-109) contienen el otro ter- 


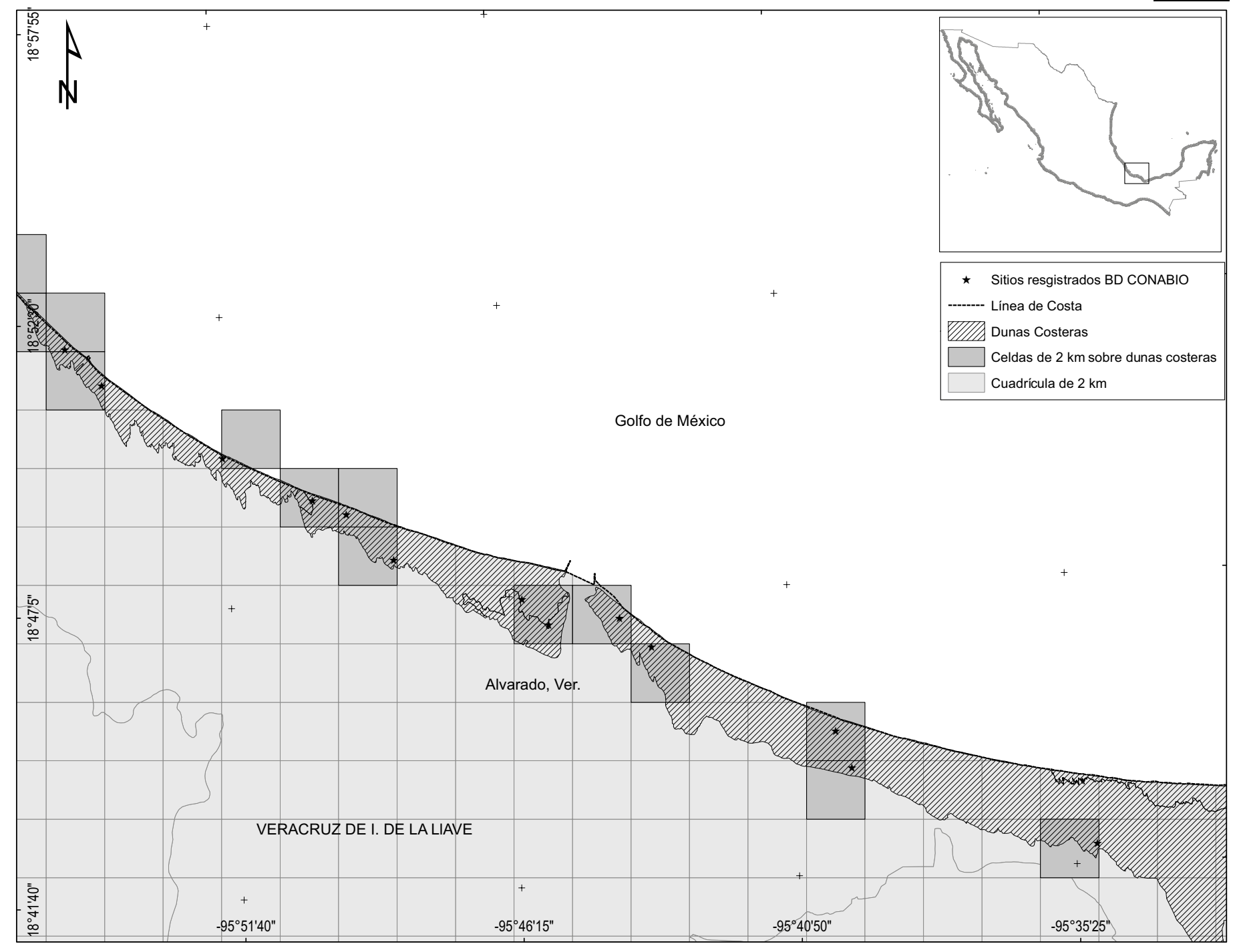

Figura 1: Franja costera dividida en celdas de $2 \times 2 \mathrm{~km}$ (ancho promedio de las dunas de México de Jiménez-Orocio et al. (2014a). Se muestra un ejemplo de celda con los puntos de localización (sitios) de cada registro.

cio (34\%) de las especies que se encuentran en playas y dunas costeras de México. Los géneros con más especies son Euphorbia (44), Cyperus (30), Atriplex (19), Ipomoea (18), Croton y Passiflora (16), y finalmente, Ambrosia y Bursera (15).

\section{Distribución por colecciones}

Los valores altos de IR indican que las especies colectadas tienen un número alto de especímenes por especie. Si el número de especies es alto, quiere decir que tienen muchas especies y muchos registros por cada especie.
Este es el escenario ideal para una colección. Las cuatro colecciones nacionales con índices de redundancia mayores o iguales a 0.7 (Fig. 3) corresponden a los herbarios de la Universidad Nacional Autónoma de México (MEXU, IR=0.9), el Centro de Investigaciones Científicas de Yucatán (CICY, IR=0.8) que cubre básicamente la flora de playas y dunas costeras del Caribe mexicano en la costas de los estados de la Península de Yucatán (Campeche, Yucatán y Quintana Roo) y contiene las dos principales colecciones que se han hecho en esa región, una en los años 1980 y la que corresponde al proyecto 


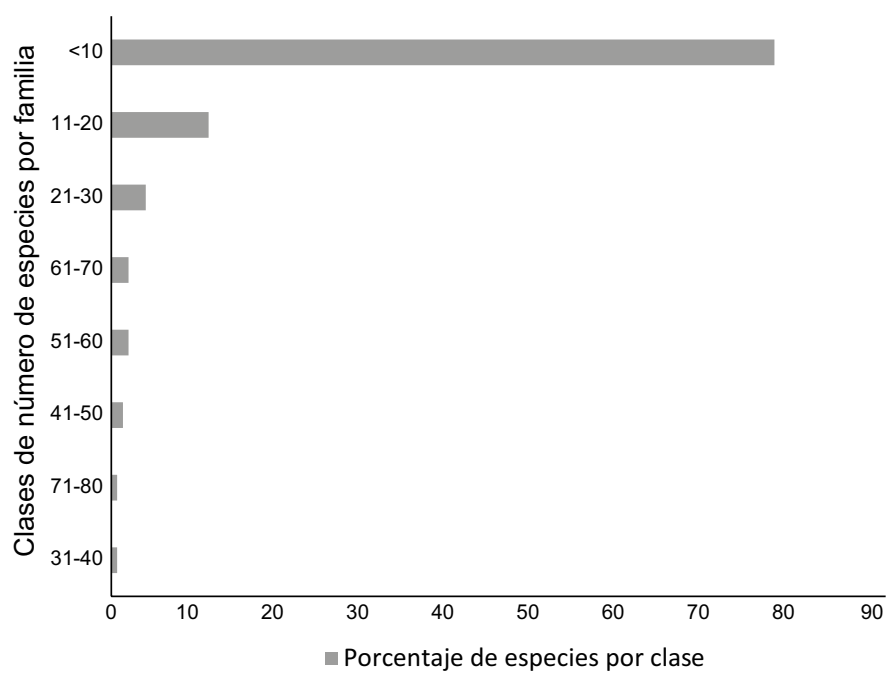

Figura 2: Distribución taxónomica de las especies por familias de la flora en playas y dunas de México. No están graficadas las cuatro familias con más especies: Asteraceae con 248 especies, Fabaceae con 223 especies, Poaceae con 168 especies y Euphorbiaceae con 109 especies.

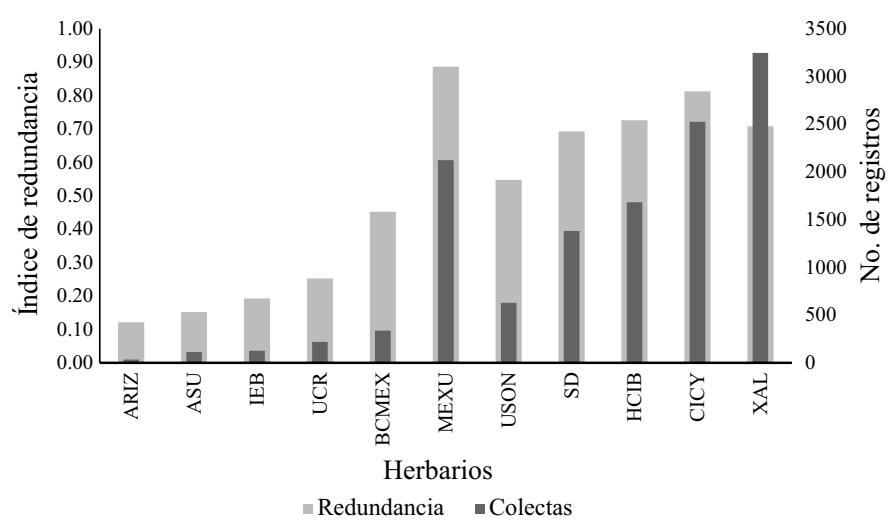

Figura 3: Especies y registros de colectas e índice de redundancia (IR) de las colecciones con plantas en playas y dunas costeras de México.

de CONABIO. La colección del Centro de Investigaciones Biológicas del Noroeste (HCIB, IR=0.7) cubre casi por completo las playas y dunas de Baja California Sur (aunque faltan colectas en las dunas del norte del estado), pero representa la flora de playas y dunas costeras del Pacífico mexicano y del Golfo de California. En el herbario del Instituto de Ecología, A.C. (XAL, IR=0.7) se encuentran las colecciones botánicas del litoral del
Golfo de México de las playas y dunas costeras de Tamaulipas, Veracruz, Jalisco y Oaxaca, principalmente. La colección internacional con mejor esfuerzo de colecta está en el Museo de Historia Natural de San Diego ( $\mathrm{SD}, \mathrm{IR}=0.7$ ) que cubre la flora de la Península de Baja California (Baja California y Baja California Sur). Si el número de especies es bajo, la colección tiene muchas colectas, pero de pocas especies. Por ejemplo, la colección de la Universidad de Sonora (USON, IR=0.55) hizo un esfuerzo de colecta excepcional para este proyecto, pero no tiene colecciones históricas y por lo tanto no tiene muchos ejemplares de las especies en las playas y dunas del estado.

Si el número de especies es bajo, correspondería al peor de los escenarios tanto para colector como para colección. Si el número de especies es alto, quiere decir que hay muchas especies y pocos ejemplares por cada especie.

En el caso de colectores indica que ha maximizado la colección (de especies nuevas) en su esfuerzo de colecta. Este sería el escenario ideal de un colector. De los 556 colectores registrados, Gonzalo Castillo del INECOL (XAL) sobresale por mucho habiendo colectado $12 \%$ del total de los ejemplares (1502) y la tercera parte $(30.6 \%)$ de las especies (610) $(\mathrm{IR}=0.59)$ en playas y dunas de México. Sus colectas especialmente son del Golfo de México y también ha colectado en el Pacífico Sur. Merari Ferrer colectó 779 ejemplares de 172 especies $(\mathrm{IR}=0.78)$ de la Península de Yucatán para el proyecto de CONABIO. Esta proporción de muchos ejemplares de relativamente pocas especies resulta en un $\mathrm{IR}=0.8$, el más alto de todos los colectores. Le siguen en número de colectas, botánicos de la Península de Baja California, Reid Moran (SD) con 549 ejemplares de 261 especies ( $\mathrm{IR}=0.52$ ), José Juan Pérez Navarro (HCIB) con 530 ejemplares de 188 especies $(\mathrm{IR}=0.64)$ y José Luis León de la Luz (HCIB) con 433 ejemplares de 229 especies ( $I R=0.47)$. Otros 12 colectores tienen rangos mayores a 200 ejemplares y a 100 especies y son tanto conocidos taxónomos y ecólogos regionales, como colectores aficionados o autores de tesis y artículos referentes a la vegetación local en playas y du- 
nas costeras: Edgar Cabrera, Cástulo Chan, José Salvador Flores y Rafael Durán en la Península de Yucatán; Jon Rebman en la Península de Baja California; Miguel Domínguez y Debra Valvov en Baja California Sur; Ileana Espejel en la Península de Yucatán y en Baja California; Patricia Moreno-Casasola en las dunas del Golfo de México, Caribe y Pacífico Sur; Hugo López y Verónica Espejel en el Pacífico Sur y José de Jesús Sánchez y Richard Felger en Sonora.

\section{Regiones florísticas de las playas y dunas costeras de México}

La franja de costa con playas y dunas costeras cubre una superficie de $266,448 \mathrm{~km}^{2}$ dentro de la cual hay 999 celdas de $4 \mathrm{~km}^{2}$ con colectas en playas y dunas costeras, distribuidas en los 17 estados costeros de México. Se identifi- caron 2180 sitios con alguna colecta de plantas vasculares en playas y dunas costeras.

En la figura 4 se muestran los resultados del análisis de conglomerados por especies y localidades, las agrupaciones se relacionaron con variables geográficas y políticas. Se encontró una clara concordancia con las principales regiones marinas de México: Pacífico Norte, Golfo de California, Pacífico Sur, Golfo de México y Mar Caribe.

Las regiones florísticas con más alta diversidad de plantas vasculares en playas y dunas son las denominadas Golfo de México y Golfo de California y la región florística de menor diversidad es la Pacífico Sur (Fig. 5).

\section{La flora en playas y dunas de México}

Según la base de datos, las primeras especies colectadas en las dunas costeras de México no son estrictas de du-

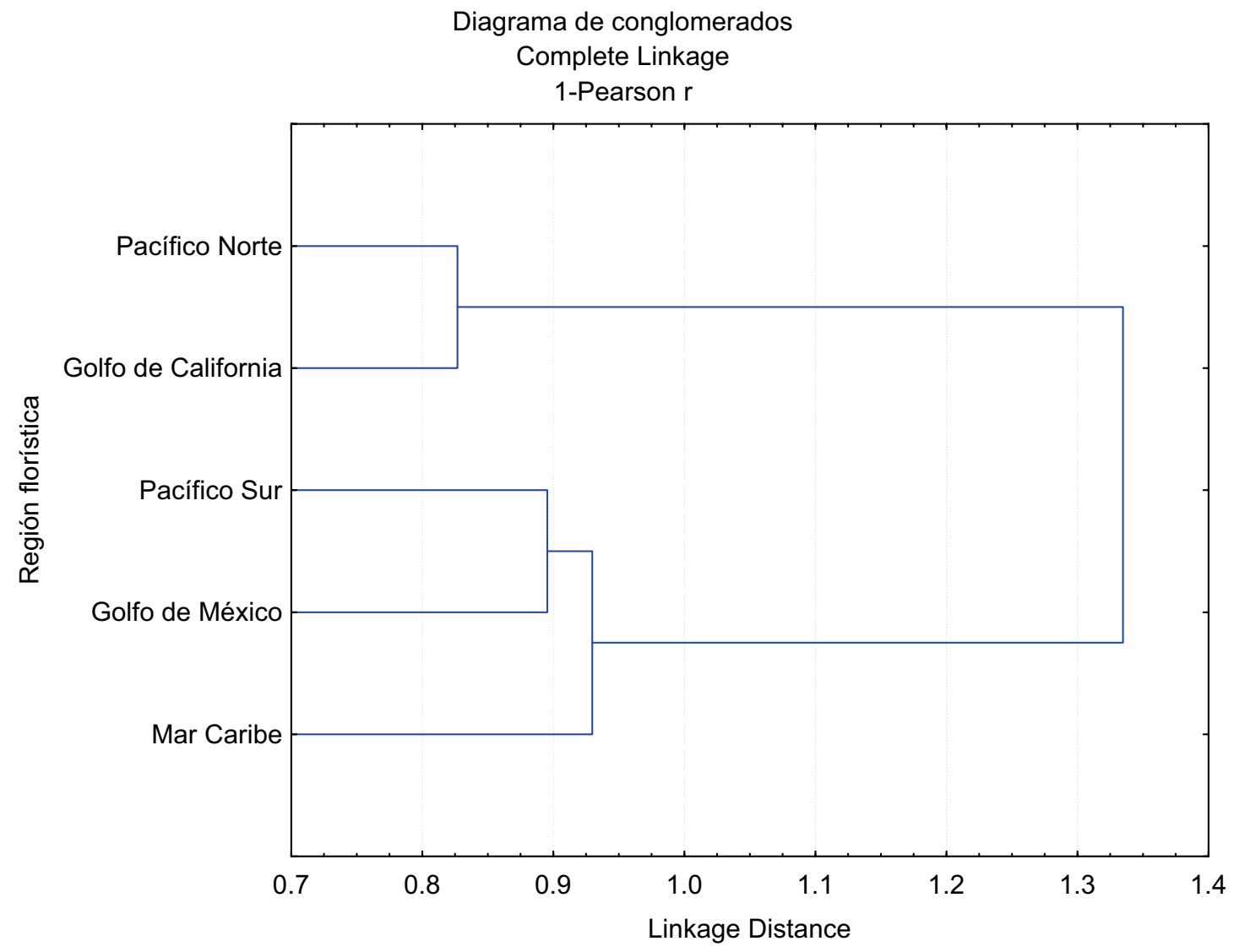

Figura 4: Dendrograma que muestra las cinco regiones florísticas de las playas y dunas costeras de México. El análisis se hizo por municipios, pero por cuestiones de claridad en la imagen, aquí se presenta simplificado. 


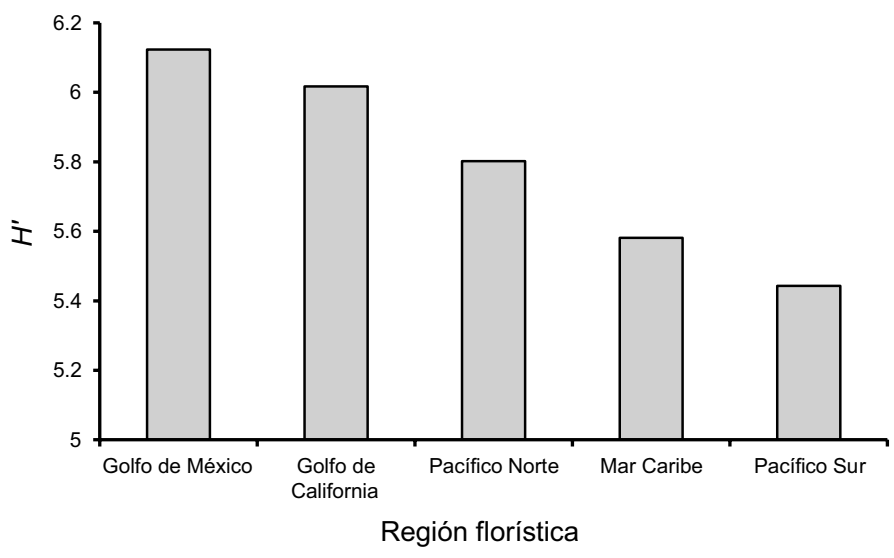

Figura 5: Índice de diversidad de Shannon $\left(H^{\prime}\right)$ para comparar las cinco regiones. Las varianzas son Golfo de México $=0.00048$, Golfo de California $=0.00043$, Pacífico Norte $=0.00055$, Mar Caribe $=0.00045$ y Pacífico Sur $=0.0017$.

nas (Aesculus parryi A. Gray, Eriogonum repens Reveal, Aristida adscenionis L.). Es hasta 1929 que I. L. Wiggins colectó la primera planta estricta de dunas costeras (Fig. 6), Atriplex leucophylla (Moq.) D. Dietr., en la desembo- cadura del arroyo Guadalupe en la playa de La Misión al norte de Ensenada, Baja California y ha sido colectada 12 veces más en Eréndira y El Rosario, en Baja California y en Isla Magdalena, Baja California Sur, entre otros lugares.

Al analizar la intensidad de las colectas a nivel nacional, se distinguen tres etapas: I) inicial, de crecimiento más o menos intenso, o con fases de mayor intensidad, que correspondería a los años previos a 1990-92, en el caso del Mar Caribe (CAR) es un poco antes (1985-87); II) media, ralentización del crecimiento de la tendencia, 1992-2010 y III) final, breve de incremento, 2010-12.

\section{Discusión}

Esta investigación actualiza el conocimiento de la flora en dunas costeras de México el cual se encontraba incompleto, desarticulado y desactualizado. Algunos estudios han actualizado listados florísticos de una región, por ejemplo, el trabajo de Torres et al. (2010) actualizó los listados que Espejel (1986) y Moreno-Casasola y Espejel (1986)

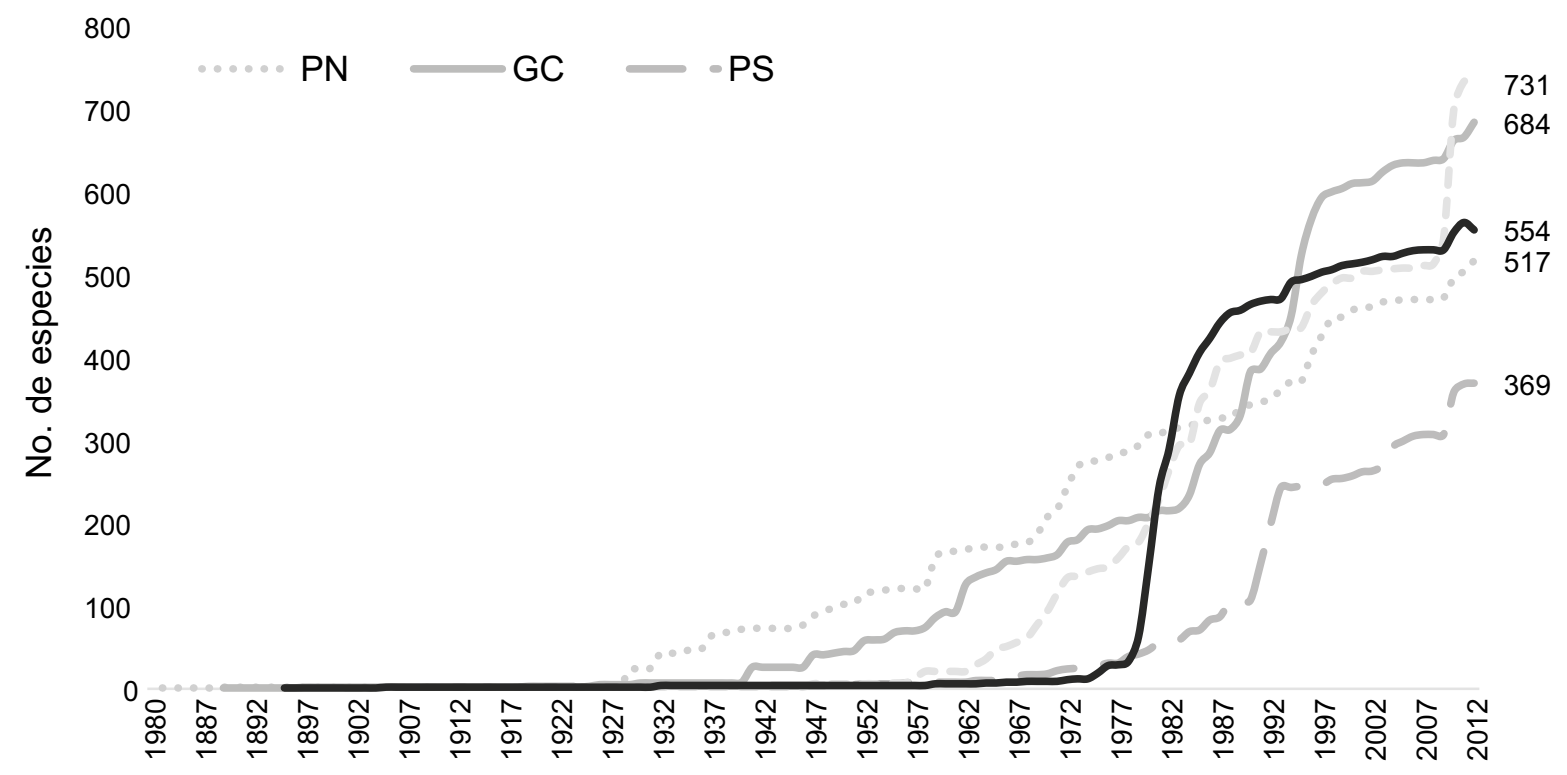

Año de colecta

Figura 6: Acumulación temporal del número de especies y del número de colectas de la flora de playas y dunas de México entre 1883 y 2012. $\mathrm{GC}=$ Golfo de California (684 especies), PN=Pacífico Norte (517 especies), PS=Pacífico Sur (369 especies), GM=Golfo de México (732 especies) y CAR=Mar Caribe (554 especies). Los cambios en las pendientes significan el aumento en el esfuerzo de colecta producto de proyectos en dunas costeras como tesis de doctorado y del proyecto CONABIO (Espejel et al., 2015). 
publicaron sobre las dunas de Yucatán el siglo pasado. Otros estudios concentran y actualizan listados regionales como Vanderplank (2011) para San Quintín, y Harper et al. (2011) para Colonet, en Baja California (zonas costeras incluidas en el trabajo de Johnson (1977)). Hay otros sitios que constantemente han sido colectados y permanentemente se actualizan los listados; ejemplo especial son las dunas de la estación biológica de La Mancha, Veracruz (Moreno-Casasola, 1982) y que Jiménez-Orocio et al. (2015) reconocen como las dunas costeras cuya flora ha sido más colectada del país.

Enmarcar las localidades de colecta de los 12,419 registros en las celdas de $4 \mathrm{~km}^{2}$ permitió visualizar una colecta relativamente homogénea en todo el país. Sin embargo, permanecen sitios sin colectas suficientes; especialmente llamativas son las playas y dunas de las costas del Pacífico Sur donde se desarrolla selva baja caducifolia sobre dunas (Martínez et al., 2014), al igual que en Veracruz donde además hay bosque de encino, selva de chicozapote y selva de ébano (Castillo et al., 2016). Los estados del Pacífico Sur requieren un proyecto particular sobre riqueza florística en dunas costeras para perfeccionar el estudio de país.

\section{Sobre las colecciones y los colectores}

Las colecciónes más completas son las de MEXU y XAL, tienen a las personas que más han colectado en las playas y dunas costeras. Es importante citar esto porque hay especialistas de la flora de familias y de sitios, pero no es común entre los colectores que se hable de expertos en un tipo de vegetación determinado. Para la gráfica de la Figura 3 se eliminaron los herbarios con pocas especies (menos de 10) e índices de redundancia altos, porque sus objetivos no son la flora de México (ARIZ, CAVA, DES, etc.) (Cuadro), aunque aportan registros interesantes para el noroeste del país.

Las especializaciones regionales de los herbarios son: 1) en XAL prevalecen las floras en playas y dunas costeras del Golfo de México y algunos estados del Pacífico Sur; 2) CICY contiene completamente a la flora de la Península de Yucatán y 3) las regiones del Golfo de Ca- lifornia y Pacífico Norte están bien representadas en tres colecciones: USON, HCIB y BCMEX, en México. La flora en playas y dunas costeras de Península de Baja California se encuentra sobre todo en SD en California, EUA, en el proyecto Bajaflora (http://bajaflora.org) que conjunta las colecciones de BCMEX, HCIB, RSA, SBBG, SD y UCR) y las mantiene actualizadas. El Pacífico Sur requiere de un herbario regional tan sólido como los analizados en este trabajo. La mejor colección de esta región está en el Herbario IEB del INECOL, Centro Regional del Bajío con duplicados en XAL (Cuadro).

Las cuatro etapas de colecta en playas y dunas costeras de México obedecen a la instalación de instituciones grandes en las regiones; por ejemplo, la llegada de Instituto Nacional de Investigación sobre Recursos Bioticos (INIREB) a Xalapa y del Instituto de Ecología, A.C. (INECOL) a esta misma ciudad en Veracruz, del proyecto de dunas costeras del Laboratorio de Ecología de la Facultad de Ciencias (UNAM) depositadas tanto en MEXU como en XAL, y el proyecto Etnoflora Yucatanense en la Península de Yucatán son la causa de un aumento de colectas en la década de 1980. También en la Península de Baja California, la llegada de botánicos y ecólogos vegetales al Centro de Investigaciones Biológicas del Noroeste (CIBNOR) y a la Universidad Autónoma de Baja California (IUABC) y el trabajo intenso del Museo de Historia Natural de San Diego a través de su proyecto Bajaflora explican el incremento de colectas en la década de 1990. En esta época la participación de instituciones académicas, como el Instituto de Ecología, A.C., en estudios de ordenamiento territorial de zonas costeras dio lugar a colectas en Jalisco, Guerrero y Oaxaca. Finalmente, los resultados del esfuerzo de colecta del presente trabajo se reflejan entre 2010 y 2012, donde se agregan registros de las especies de las selvas sobre dunas costeras que no se habían reportado en Veracruz, Tamaulipas, Oaxaca y Chiapas; se hace una colección botánica de las dunas de Sonora donde había muy pocos ejemplares y se suman los herbarios de Arizona que se citan en el Cuadro.

Asimismo, hay incorporaciones recientes que deben adicionarse a la base de datos en el futuro, como son 
las colectas de Sula Vanderplank en su tesis doctoral y otras publicaciones (Vanderplank et al., 2011), cuyos ejemplares no se encontraban en la base de datos del Rancho Santa Anna (RSA) cuando se hizo esta investigación, aunque están consideradas como registros bibliográficos en la base de datos de CONABIO. Los ejemplares de MEXU, que no pudieron incorporarse por falta de los campos de información obligatorios según las reglamentaciones de CONABIO, sí fueron incorporados en el listado que aquí se analiza.

Existen otros herbarios que tienen ejemplares y no fueron consultados como es el Botanical Research Institute of Texas y quizás en el estado de Florida (USA) también se tengan ejemplares de Tamaulipas y Yucatán, respectivamente. Para completar este listado habría que revisar más herbarios extranjeros. Herbarios que recientemente han crecido y posiblemente hayan incrementado sus listados de flora de dunas, como es el de la Universidad Juárez Autónoma de Tabasco (UJAT) o el de la Sociedad para el Estudio de los Recursos Bióticos de Oaxaca (SERBO) en Oaxaca, también deben consultarse en una actualización a este listado.

Un IR bajo puede significar que tenga una gran limitación de espacio y priorice tener muchas especies y no acepte nuevos especímenes de especies ya presentes en la colección. En la práctica esto último suele suceder con la mayoría de las colecciones de los estados y extranjeras.

\section{Sobre las regiones florísticas de las dunas costeras de México}

En una primera aproximación, Moreno-Casasola et al. (1998) distinguieron las cinco regiones que se confirman estadísticamente en este análisis. En el dendrograma de la Figura 4, las regiones del noroeste se separan del resto debido a la influencia de las floras de los desiertos, mientras que en las otras tres regiones, la influencia es de especies de las selvas tropicales. Sin embargo, las playas y dunas costeras con mayor diversidad son las que corresponden a los dos Golfos, el de México y el de California. Esto puede explicarse porque en las dunas de Veracruz y Tabasco crecen selvas con una alta diversidad y en el
Golfo de California convergen floras de varios tipos de desiertos al norte y de las selvas tropicales de Sinaloa al sur (Johnson, 1995). Asimismo, en el Pacífico Norte se desarrolla una flora que combina vegetación del desierto central con la vegetación tipo mediterráneo al norte (Peinado et al., 2011) y selvas tropicales al sur (Peinado et al., 1994). La composición florística en las playas y dunas costeras tiene tantos elementos de la vegetación circundante que el análisis particular de las floras regionales es motivo de publicaciones focales y seguramente surgirán sub-regionalizaciones. Asimismo, merece un análisis más detallado de las 95 especies consideradas como preferentes de playas y dunas.

En los capítulos del Diagnóstico General de las Dunas Costeras de México (Martínez et al., 2014) que se refieren a los estados (Moreno-Casasola et al., 2014a,b,c,d; Jiménez-Orocio et al., 2014a,b,c), se mencionan distintos grados de perturbación en la vegetación de dunas y es común que en los herbarios no haya colectas de la flora exótica ni de especies cultivadas como son cocos, melones, papayas, etc. En el caso de las playas y dunas sería interesante volver a colectar en aquellos sitios bien estudiados en la década de los 1980, para observar los efectos que los cambios experimentados debido a ganadería o desarrollo habitacional han tenido sobre la flora.

Cabe mencionar que aún hay poca información sobre la flora de dunas costeras en las islas. Algunas son cayos arenosos, sobre todo en zonas arrecifales, mientras otras tienen mayor variedad de suelos y tipos de vegetación, como las Islas Revillagigedo. Su composición florística es de gran interés para estudiar ya que permite entender cuáles especies son capaces de dispersarse a mayores distancias y pueden colonizar arenas móviles, además de que en las islas hay menor disponibilidad de agua dulce que en el continente. Asimismo, se producen situaciones fitogeográficas interesantes, por ejemplo, la vegetación de las islas del sistema arrecifal veracruzano tiene especies comunes con la flora de dunas de la Península de Yucatán, por estar formadas por arena calcárea, a diferencia de las dunas de tierra firme de dicho estado, formadas por arenas silíceas, que tienen una flora muy distinta (Moreno-Casasola et al., 1998). 


\section{Sobre la flora}

De las especies registradas solo se definen 95 especies como preferentes de playas y dunas costeras. Esta baja proporción de especies (4.5\%) refleja que son pocas las especies especializadas para las condiciones tan extremas de los ambientes de playas y dunas móviles (grandes movimientos de arena, salinidad en suelo y aire y altas temperaturas) (Castillo y Moreno-Casasola, 1996). Sin embargo, quizá también el escaso número de especies especializadas en estos sistemas tiene que ver con la alta variabilidad de los mismos (aceptando que la estabilidad está directamente asociada con la riqueza de especies). Sin embargo, que en las dunas se compartan 1978 especies de otros tipos de vegetación evidencia el carácter de ecotono que caracteriza a estos ecosistemas (Peinado et al., 2011).

La flora en playas y dunas costeras representa $9.5 \%$ de las plantas vasculares de México ya que Villaseñor y Ortíz (2014) mencionan que en el país hay cerca de 21,841 especies. La proporción también se explica por la presencia de distintos microambientes dados por la microtopografía que caracteriza a las dunas costeras en el mundo y en México (Moreno-Casasola, 1986; Alpert y Mooney, 1996; Álvarez-Rogel, 2007 entre otros): existen zonas inundadas (lagunas interdunarias) y zonas con escasez de agua y vientos fuertes (crestas), zonas protegidas del viento y aspersión salina (laderas barlovento) y zonas expuestas a fuertes vientos y aspersión salina (ladera sotavento), zonas pobres en nutrientes (crestas) y zonas con mayor fertilidad (hondonadas húmedas). Lo anterior aunado a su distribución a lo largo del gradiente climático que caracteriza a las costas mexicanas, el cual permite que las dunas sean hábitats apropiados para este número de especies.

Las 153 familias registradas representan más de la mitad (62\%) del total de las familias de plantas vasculares mexicanas (247 según Villaseñor y Ortíz, 2014). Como en todo el país, las familias de plantas en playas y dunas costeras con más registros son Asteraceae, Fabaceae y Poaceae (Villaseñor y Ortiz, 2014). En estos ecosistemas, casi la tercera parte de las especies (601 especies) corresponden a estas tres familias (Moreno-Casasola et al., 1998).
La familia Euphorbiaceae es una de las familias mejor representadas en México (con 43 géneros y 782 especies (Villaseñor, 2004)) y lo es también en las playas y dunas costeras, con la mitad de los géneros de esta familia (24) y 109 especies. En la Figura 2 se muestra el resto de la distribución de las especies en las 149 familias restantes. Las familias con menos de 10 especies dominan por mucho la flora en las playas y dunas costeras de México.

Los 897 géneros de las playas y dunas mexicanas representan la tercera parte $(33.4 \%)$ de los géneros de plantas vasculares de México (2285 según Villaseñor y Ortíz, 2014). El género Euphorbia es el que más especies tiene en México (Steinmann, 2002) y en las playas y dunas también es el que presenta mayor cantidad de especies.

Las especies con más registros son Trianthema portulacastrum L. (165 registros) cuya sinonimia más conocida es Sesuvium portulacastrum (L.) L. y cuyo nombre común es "verdolaga de playa". Es una especie considerada como maleza (Villaseñor y Espinoza, 1998) y muy común en todas las playas mexicanas, por lo que se explica que es la especie más colectada. Croton punctatus Jacq. (107) es una hierba común en las playas y dunas tropicales de México, como florece casi todo el año es posible que sea la razón de su amplia colecta. Echites umbellatus Jacq. (106) es común en las costas del Golfo de México y el Caribe, florece casi todo el año y sus flores blancas y frutos rojos típicos de Apocynaceae son muy llamativos y quizás eso explica su amplia colecta. Finalmente, Ipomoea pes-caprae (90) es la especie pionera más común en las playas de México (con excepción del noroeste del país). Es posible que sus flores llamativas y uso medicinal (Devall, 1992) hayan motivado la extensa colección de la especie. Sus flores lilas-moradas, junto con las flores del mismo color de Canavalia rosea (Sw.) DC. (87) son muy llamativas. Son las hierbas, rastreras y de hojas semi-suculentas, con carácter pantropical, típicas colonizadoras de dunas. Ambas son de las principales especies que forman dunas embrionarias y estabilizan el primer cordón de dunas en las playas del sur de México (Castillo y Moreno-Casasola, 1996). 
Otras especies muy colectadas son Amaranthus greggii S. Watson (84) que es un arbusto pequeño muy común en las dunas costeras del Golfo de México. Oenothera drummondii Hook. (83) es una onagrácea de flor amarilla muy llamativa que domina las costas de Baja California Sur, aunque también se encuentra en el Golfo de México. Las palmas que dominan las costas de Yucatán y Quintana Roo, Coccothrinax argentata (Jacq.) L.H. Bailey (80) y Thrinax radiata Lodd. ex Schult. \& Schult. f. (72), son de distribución caribeña y han sido muy colectadas por su accesibilidad y valor ornamental. El pasto más común en las playas y dunas es Sporobolus virginicus (L.) Kunth (72) y porque ocupa grandes superficies de dunas que han sido transformadas en potreros, especialmente en Veracruz. Además, es una de las gramíneas que toleran condiciones de salinidad y movimiento de arena en la orilla de la playa, formando dunas embrionarias, así como condiciones de inundación temporal formando parte de las depresiones (Moreno-Casasola et al., 2014e). Otra especie muy colectada es Scaevola plumieri (L.) Vahl (71 registros), arbusto cosmopolita dominante en las playas sudafricanas (Knevel y Lubke, 2005) y abundante en la Península de Yucatán y Pacífico Sur, donde forma dunas embrionarias. Su flor pequeña y asimétrica es llamativa y posiblemente eso haya motivado la abundancia de colectas.

Un problema persistente en este tipo de trabajos es la nomenclatura. Las sinonimias se resolvieron en Tropicos.org (TROPICOS, 2015) y Villaseñor y Ortíz (2014), pero los cambios en nomenclatura son continuos conforme avanzan los estudios taxonómicos y los análisis genéticos.

\section{CONCLUSIONES}

El listado de plantas vasculares que aquí se presenta forma el primer inventario florístico en las playas y dunas costeras de México, georreferenciado y respaldado con ejemplares de herbario.

La magnitud de la flora en playas y dunas del país es de 153 familias, 897 géneros y 2076 especies de plantas vasculares, las cuales representan $9.5 \%$ de la flora vascular de México. Este porcentaje no es despreciable dado que en general las playas y dunas costeras ocupan una angosta franja del país ( $2 \mathrm{~km}$ en promedio) como calcularon Jiménez-Orocio et al. (2014a). Las especies con más registros son las consideradas "típicas" de playas y dunas (Trianthema portulacastrum, Croton punctatus, Echites umbellatus e Ipomoea pes-caprae) y son parte de las 95 especies preferentes de estos ambientes. Por el carácter de ecotono de las dunas costeras y su variabilidad de tipos distintos de ambientes, el resto de las especies de plantas (1981) se comparten con los tipos de vegetación vecinos (selvas, matorrales, humedales de agua dulce, marismas y manglares). Asimismo, hay registros de herbario de plantas escapadas de cultivos como Carica papaya L., Cucumis melo L. o cultivos importantes como es Cocos nucifera L.

Es importante mencionar que durante el trabajo de campo hemos observado especies de cactáceas, árboles tropicales y arbustos del desierto que no han sido incorporadas a herbarios. Por otro lado, la base de datos debe completarse con datos de sus estatus de conservación real, no solo de aquellas que están en la NOM-059-SEMARNAT-2010 (SEMARNAT, 2010), ya que la deforestación y fragmentación cerca de las ciudades o en nuevos desarrollos turísticos es motivo de pérdida de biodiversidad en las playas y dunas costeras en México (Mendoza-González et al., 2012) como en otras partes del mundo (Heslenfeld et al., 2004).

El principal colector de estos sistemas es Gonzalo Castillo del INECOL, quien ha colectado especialmente en las playas y dunas del Golfo de México y Pacífico Sur.

Las colecciones regionales con más especies y colectas de estos ecosistemas son los herbarios de la Universidad Nacional Autónoma de México, del Centro de Investigación Científica de Yucatán, del Centro de Investigaciones Biológicas del Noroeste y del Instituto de Ecología, A.C. De las colecciones extranjeras revisadas, la del Museo de Historia Natural de San Diego es un referente necesario para la Península de Baja California y/o las regiones florísticas Pacífico Norte y Golfo de California. El herbario de la Universidad de Sonora realizó un esfuerzo muy importante para completar su flora estatal a través del proyecto de CONABIO. Los resultados sugieren fortalecer algún herbario en el Pacífico Sur. 
Los sitios que se han colectado mejor son el sur de la Península de Baja California y el estado de Veracruz. Es necesario revisar los herbarios del Pacífico Sur (colectas enteras de vegetación costera se perdieron por algún desastre en herbarios) (Saturnino Díaz, com. pers.). Asimismo, es necesario revisar nuevamente los herbarios nacionales como MEXU y los regionales que no fueron incluidos y que recientemente se han digitalizado como el de la Universidad Autónoma de Sinaloa (UAS) (VegaAviña, 2015). La investigación necesaria consta de revisar ejemplares etiquetados en selvas bajas y medianas en localidades costeras del Pacífico Sur y relacionarlas con el mapa de distribución de dunas costeras de esos estados. Además, es importante incrementar el trabajo de campo y las colectas en esos estados para completar las colecciones y que tengan el mismo esfuerzo de colecta que las otras regiones estudiadas (para que al menos todas tengan un $\mathrm{IR}=0.7$ ).

La variación espacial de las especies de plantas en un área geográfica tan grande y heterogénea como es el litoral mexicano se ajusta en un primer análisis a cinco regiones florísticas que corresponden claramente a los mares de México, de ahí que así se nombraron. La región Pacífico Norte tiene una mayor diversidad que el Pacífico Sur. Un estudio importante para realizar en el futuro es la comparación de las floras en términos biogeográficos y comparaciones con las floras vecinas, lo cual también podría ayudar a comprender las diversidades diferenciadas de esta flora. Asimismo, serán interesantes los estudios regionales para entender cada región florística independientemente de, o en relación a las otras.

Esta primera lista de la flora en playas y dunas es la base para múltiples estudios florísticos, biogeográficos y ecológicos y será importante para su uso en temas de impacto ambiental y manejo integrado de la zona costera como se sugiere en Pedroza et al. (2013).

\section{CONTRIBUCIONES DE LOS AUTORES}

IE, OJ, PG, GC, RD, MF, DI, JL, HL, AM, PM, NR y JS contribuyeron en la revisión de herbarios y la colecta de ejemplares en campo de diferentes playas y dunas del país. Asimismo, colaboraron en la redacción, revisión del texto y del listado florístico. LA, RM y OJ colaboraron en los análisis. SC y SI colaboraron en la revisión del herbario MEXU y revisión del documento final. JR y SV colaboraron en la revisión del listado florístico y traducción del resumen en inglés.

\section{FINANCIAMIENTO}

Este estudio fue apoyado por el proyecto "Flora de Playas y Dunas de México"; financiado por la Comisión Nacional para el Conocimiento y Uso de la BiodiversidadCONABIO (HJ007).

\section{AgRAdECIMIENTOS}

En los grupos de trabajo participaron en total 56 personas entre técnicos, estudiantes y voluntarios, a todos ellos un reconocimiento especial. Se agradece especialmente a María Elena Medina Abreo por su apoyo en las múltiples revisiones del listado florístico. Asimismo, agradecemos a dos revisores anónimos que ayudaron a mejorar el artículo.

\section{LITERATURA CITADA}

Alpert, P. y H. A. Mooney. 1996. Resource heterogeneity generated by shrubs and topography on coastal sand dunes. Vegetatio 122(1): 83-93. DOI: http://dx.doi. org/10.1007/BF00052818

Álvarez-Rogel, J., L. Carrasco, C. M. Marín y J. J. MartínezSánchez. 2007. Soils of a dune coastal salt marsh system in relation to groundwater level, micro-topography and vegetation under a semiarid Mediterranean climate in SE Spain. Catena 69(2): 111-121. DOI: http://dx.doi. org/10.1016/j.catena.2006.04.024

Álvarez-Molina, L. L., M. L. Martínez, O. Pérez-Maqueo, J. B. Gallego-Fernández y P. Flores. 2012. Richness, diversity, and rate of primary succession over 20 year in tropical coastal dunes. Plant Ecology 213(10): 1597-1608. DOI: http://dx.doi.org/10.1007/s11258-012-0114-5

Castillo, S., J. Popma y P. Moreno-Casasola. 1991. Coastal sand dune vegetation of Tabasco and Campeche, Mexico. Journal of Vegetation Science 2(1): 73-88. DOI: http:// dx.doi.org/10.2307/3235899 
Castillo, S. y P. Moreno-Casasola. 1996. Coastal sand dune vegetation: an extreme case of species invasion. Journal of Coastal Conservation 2(1): 13-22. DOI: http://dx.doi. org/10.1007/BF02743033

Castillo-Campos, G., P. Moreno-Casasola y J. Laborde Dovali. 2016. Bosques y selvas en las dunas. In: Moreno-Casasola (ed.). Servicios Ecosistémicos de las Selvas y Bosques Costeros de Veracruz. Instituto de Ecología, A.C. Xalapa, México. Pp. 60-75.

Castillo-Campos, G. y A. C. Travieso-Bello. 2006. La flora. In: Moreno-Casasola, P. (ed.). Entornos veracruzanos: la costa de La Mancha. Instituto de Ecología, A.C. Xalapa, México. Pp. 171-204.

Cavazos, T., J. A. Salinas, B. Martínez, G. Colorado, P. de Grau, R. Prieto-González, A. C. Conde-Álvarez, A. Quintanar-Isaías, J. S. Santana-Sepúlveda, R. Romero-Centeno, M. E. MayaMagaña, J. G. Rosario de la Cruz, M. R. Ayala-Enríquez, H. Carrillo-Tlazazanatza, O. Santiesteban y M. E. Bravo. 2013. Actualización de escenarios de cambio climático para México como parte de los productos de la quinta comunicación nacional. Informe Final del Proyecto al INECC. México, D. F., México. 150 pp. http://www.inecc.gob.mx/descargas/ cclimatico/2012_estudio_cc_vyagef12.pdf

CONABIO. 2009. Catálogo taxonómico de especies de México. In: Soberón, J., G. Halffter y J. LlorenteBousquets (comps.). Capital Natural de México, Vol. I: Conocimiento actual de la biodiversidad. Comisión Nacional para el Conocimiento y uso de la Biodiversidad. CD series, CD-1.

CONABIO. 2015. Sistema Integrado de Información Taxonómica SIIT http://siit.conabio.gob.mx/ y que corresponde a la versión en español de Integrated Taxonomic Information System (ITIS) base de datos en línea http://www.itis.gov (consultado julio de 2015)

Colwell, R. K. 2013. EstimateS, Version 9.1: Statistical estimation of species richness and shared species from samples (Software and User's Guide).

Derraik, J. G. 2002. The pollution of the marine environment by plastic debris: a review. Marine Pollution Bulletin 44(9): 842-852. DOI: http://dx.doi.org/10.1016/S0025$326 \mathrm{X}(02) 00220-5$
Devall, M. S. 1992. The biological flora of coastal dunes and wetlands. 2. Ipomoea pes-caprae (L.) Roth. Journal of Coastal Research 8(2): 442-456.

Devall, M. S. y L. B. Thien. 2005. Inland occurrence of the strand plant Ipomoea pes-caprae (Convolvulaceae) around Lake Nicaragua. The Southwestern Naturalist 50(3): 380-384. DOI: http://dx.doi.org/10.1894/00384909(2005)050[0380:IOOTSP]2.0.CO;2

Espejel, I. 1986. Vegetación de las dunas costeras de la Península de Yucatán. II. Reserva de la biosfera Sian Ka'an, Quintana Roo, México. Biótica 11(1): 7-14.

Espejel, I. 1987. A phytogeographical analysis of coastal vegetation in the Yucatán Peninsula. Journal of Biogeography 14(6): 499-519. DOI: http://dx.doi.org/10.2307/2844877

Espejel, I., O. Jiménez-Orocio y P. Peña-Garcillán. 2015. Flora de las playas y dunas costeras de México. Informe final SNIB-CONABIO, proyecto No. HJ007. Universidad Autónoma de Baja California, Facultad de Ciencias. Ensenada, México. 153 pp.

Everard, M., L. Jones y B. Watts. 2010. Have we neglected the societal importance of sand dunes? An ecosystem services perspective. Aquatic Conservation Marine and Freshwater. Ecosystem 20(4): 476-487. DOI: http:// dx.doi.org/10.1002/aqc.1114

Gallego-Fernández, J. B. y M. L. Martínez. 2011. Environmental filtering and plant functional types on mexican foredunes along the Gulf of Mexico. Ecoscience 18(1): 52-62. DOI: http://dx.doi.org/10.2980/18-1-3376

García-Mora, M. R., J. B. Gallego-Fernández, A. T. Williams y F. García-Novo. 2001. A coastal dune vulnerability classification. A case study of the SW Iberian Peninsula. Journal of Coastal Research 17(4): 802-811.

Garcillán, P. P., E. Ezcurra, y H. Riemann. 2003. Distribution and species richness of woody dryland legumes in Baja California, Mexico. Journal of Vegetation Science 14(4): 475-486. DOI: http://dx.doi. org/10.1111/j.1654-1103.2003.tb02174.x

Goldsmith, V. 1989. Coastal sand dunes as geomorphological systems. Proceedings of the Royal Society of Edinburgh, Section B: Biological Sciences 96: 3-15. DOI: https://doi. org/10.1017/S0269727000010824 
Harper, A. B., S. Vanderplank, M. Dodero, S. Mata y J. Ochoa. 2011. Plants of the Colonet region, Baja California, Mexico, and a vegetation map of Colonet Mesa. Aliso 29(1): 25-42. DOI: http://dx.doi.org/10.5642/ aliso.20112901.04

Heslenfeld, P., P. D. Jungerius y J. A. Klijn. 2004. European coastal dunes: ecological values, threats, opportunities and policy development. In: Martínez, M. L. y N. P. Psuty (eds.). Coastal Dunes: Ecology and Conservation, Ecological Studies Vol. 171. Springer Berlin Heidelberg. New York, USA. Pp. 335-351. DOI: http://dx.doi. org/10.1007/978-3-540-74002-5_20

Hesp, P. A. 2004. Coastal Dunes in the Tropics and Temperate Regions: Location, Formation, Morphology and Vegetation Processes. In: Martínez, M. L. y N. P. Psuty (eds.). Coastal Dunes: Ecology and Conservation, Ecological Studies Vol. 171. Springer Berlin Heidelberg. New York, USA. Pp. 329-49. DOI: http://dx.doi. org/10.1007/978-3-540-74002-5_3

Hesp, P., M. Martínez, G. M. da Silva, N. Rodríguez-Revelo, E. Gutiérrez, A. Humanes, D. Lainez, I. Montano, V. Palacios, A. Quesada, L. Storero, G. G. Trilla y C. Trochine. 2011. Transgressive dunefield landforms and vegetation associations, Doña Juana, Veracruz, Mexico. Earth Surface Processes and Landforms 36(3): 285-295. DOI: http://dx.doi.org/10.1002/esp.2035

INEGI. 2010. Marco Geoestadístico Nacional versión 5.0 (Censo de Población y Vivienda 2010). Instituto Nacional de Estadística y Geografía. Publicado en internet http:// www.inegi.org.mx (consultado julio de 2015).

Jiménez-Orocio, O., P. Hesp, M. L. Martínez, I. Espejel y P. Moreno-Casasola. 2014a. Tipos de dunas. In: Martínez, M. L., P. Moreno-Casasola, I. Espejel, O. Jiménez-Orocio, D. Infante-Mata y N. Rodríguez-Revelo (eds.). Diagnóstico general de las dunas costeras de México. Comisión Nacional Forestal. México, D.F., México. Pp. 27-48.

Jiménez-Orocio, O., I. Espejel, M. L. Martínez, P. MorenoCasasola, D. Infante-Mata y N. Rodríguez-Revelo. 2014b. Uso de las dunas y situación actual. In: Martínez, M. L., P. Moreno-Casasola, I. Espejel, O. Jiménez-Orocio, D. Infante-Mata y N. Rodríguez-Revelo (eds.). Diagnóstico general de las dunas costeras de México. Comisión Nacional Forestal. México, D.F., México. Pp. 81-94.

Jiménez-Orocio, O., I. Espejel, P. Moreno-Casasola, D. Infante Mata, M. L. Martínez, N. Rodríguez-Revelo y R. Monroy. 2014c. Jalisco. In: Martínez, M. L., P. MorenoCasasola, I. Espejel, O. Jiménez-Orocio, D. Infante-Mata y N. Rodríguez-Revelo (eds.). Diagnóstico general de las dunas costeras de México. Comisión Nacional Forestal. México, D.F., México. Pp. 213-220.

Jiménez-Orocio, O., I. Espejel y M. L. Martínez. 2015. La investigación científica sobre dunas costeras de México: origen, evolución y retos. Revista Mexicana de Biodiversidad 86(2): 486-507. DOI: http://dx.doi. org/10.1016/j.rmb.2015.04.022

Johnson, A. F. 1982. Dune vegetation along the eastern shore of the Gulf of California. Journal of Biogeography 9(4): 317-330. DOI: http://dx.doi.org/10.2307/2844718

Johnson, A. F. 1977. A survey of the strand and dune vegetation along the Pacific and southern gulf coast of Baja California, Mexico. Journal of Biogeography 7: 83-99. DOI: http://dx.doi.org/10.2307/3038131

Johnson, A. F. 1995. Dunes vegetation of western Mexico. In: van der Maarel, E. (ed.). Ecosystems of the World 2B. Dry coastal ecosystems. Africa, America, Asia and Oceania. Elsevier. Amsterdam, Holland. Pp. 365-373.

Jones, M. L. M., H. L. Wallace, D. Norris, S. A. Brittain, S. Haria, R. E. Jones, P. M. Rhind, B. R. Reynolds y B. A. Emmett. 2004. Changes in vegetation and soil characteristics in coastal sand dunes along a gradient of atmospheric nitrogen deposition. Plant Biology 6(5): 598605. DOI: http://dx.doi.org/10.1055/s-2004-821004

Knevel, I. C. y R. A. Lubke. 2005. Reproductive phenology of Scaevola plumieri; a key colonizer of the coastal foredunes of South Africa. Plant Ecology 175(1): 137-145. DOI: http://dx.doi.org/10.1007/s11258-004$3369-7$

Lane, C., S. J. Wright, J. Roncal y J. Maschinski. 2008. Characterizing environmental gradients and their influence on vegetation zonation in a subtropical coastal sand dune system. Journal of Coastal Research 24(4A): 213-224. DOI: http://dx.doi.org/10.2112/07-0853.1 
Martínez, M. L. y P. Moreno-Casasola. 1996. Effects of burial by sand on seedling growth and survival in six tropical sand dune species from the Gulf of Mexico. Journal of Coastal Research 12(2): 406-419.

Martínez, M. L., T. Valverde y P. Moreno-Casasola. 1992. Germination response to temperature, salinity, light and depth of sowing of ten tropical dune species. Oecologia 92(3): 343-353. DOI: http://dx.doi.org/10.1007/BF00317460

Martínez, M. L., P. Moreno-Casasola y G. Vázquez. 1997. Effects of disturbance by sand movement and inundation by water on tropical dune vegetation dynamics. Canadian Journal of Botany 75(11): 2005-2014. DOI: http://dx.doi. org/10.1139/b97-912

Martínez, M., P. Moreno-Casasola, I. Espejel, O. JiménezOrocio, D. Infante-Mata y N. Rodríguez-Revelo. 2014. Diagnóstico general de las dunas costeras de México, Comisión Nacional Forestal. México, D.F., México. 359 pp.

Mendoza-González, G., M. L. Martínez, D. Lithgow, O. PérezMaqueo y P. Simonin. 2012. Land use change and its effects on the value of ecosystem services along the coast of the Gulf of Mexico. Ecological Economics 82: 23-32. DOI: http://dx.doi.org/10.1016/j.ecolecon.2012.07.018

Moreno-Casasola, P. 1982. Ecología de la vegetación de dunas costeras: factores físicos. Biótica 7: 577-602.

Moreno-Casasola, P. 1986. Sand movement as a factor in the distribution of plant communities in a coastal dune system. Vegetatio 65(2): 67-76. DOI: http://dx.doi.org/10.1007/ BF00044876

Moreno-Casasola, P. e I. Espejel. 1986. Classification and ordination of coastalsand dune vegetation along the Gulf and Caribbean Sea of Mexico. Vegetatio 66(3): 147-182. DOI: http://dx.doi.org/ 10.1007/BF00039908

Moreno-Casasola, P., I. Espejel, S. Castillo, G. CastilloCampos, R. Duran, J. J. Pérez-Navarro, J. L. León, I. Olmsted y J. Trejo-Torres. 1998. Flora de los ambientes arenosos y rocosos de las costas de México. In: Halffter, G. (ed.). La diversidad biológica de Iberoamérica. Vol. 2. Instituto de Ecología, A.C. Xalapa, México. Pp. 177-258

Moreno-Casasola, P., H. L. Rosas, D. I. Mata, L. A. Peralta, A. C. Travieso-Bello y B. G. Warner. 2009. Environmental and anthropogenic factors associated with coastal wetland differentiation in La Mancha, Veracruz, Mexico. Plant Ecology 200(1): 37-52. DOI: http://dx.doi.org/10.1007/ s11258-008-9400-7

Moreno-Casasola, P., H. López-Rosas, D. Infante Mata, I. Espejel, O. Jiménez-Orocio, M. L. Martínez, N. Rodríguez-Revelo, V. Espejel-González y R. Monroy. 2014a. Guerrero. In: Martínez, M. L., P. MorenoCasasola, I. Espejel, O. Jiménez-Orocio, D. Infante-Mata y N. Rodríguez-Revelo (eds.). Diagnóstico general de las dunas costeras de México. Comisión Nacional Forestal. México, D.F., México. Pp. 203-209.

Moreno-Casasola, P., D. Infante Mata, I. Espejel, O. JiménezOrocio, N. Rodríguez-Revelo, M. L. Martínez y R. Monroy. 2014b. Michoacán. In: Martínez, M. L., P. Moreno-Casasola, I. Espejel, O. Jiménez-Orocio, D. Infante-Mata y N. Rodríguez-Revelo (eds.). Diagnóstico general de las dunas costeras de México. Comisión Nacional Forestal. México, D.F., México. Pp. 223-230.

Moreno-Casasola, P., H. López-Rosas, D. Infante Mata, I. Espejel, O. Jiménez-Orocio, N. Rodríguez-Revelo, V. Espejel González, M. L. Martínez y R. Monroy. 2014c. Oaxaca. In: Martínez, M. L., P. Moreno-Casasola, I. Espejel, O. Jiménez-Orocio, D. Infante-Mata y N. Rodríguez-Revelo (eds.). Diagnóstico general de las dunas costeras de México. Comisión Nacional Forestal. México, D.F., México. Pp. 244-252.

Moreno-Casasola, P., D. Infante Mata H. López-Rosas, I. Espejel, O. Jiménez-Orocio, M. L. Martínez, N. Rodríguez-Revelo, V. Espejel González y R. Monroy, 2014d. Chiapas. In: Martínez, M. L., P. MorenoCasasola, I. Espejel, O. Jiménez-Orocio, D. Infante-Mata y N. Rodríguez-Revelo (eds.). Diagnóstico general de las dunas costeras de México. Comisión Nacional Forestal. México, D.F., México. Pp. 183-90.

Moreno-Casasola, P., H. López-Rosas y K. Rodríguez-Medina. 2014e. From tropical wetlands to pastures on the coast of the Gulf of Mexico. Pastos 42(2): 185-217.

Muñoz-Vallés, S., J. B. Gallego-Fernández y C. M. Dellafiore. 2011. Dune vulnerability in relation to tourism pressure in central Gulf of Cádiz (SW Spain), a case study. Journal 
of Coastal Research 27(2): 243-251. DOI: http://dx.doi. org/10.2112/JCOASTRES-D-09-00125.1

Pedroza, D., A. Cid, O. García, R. Silva-Casarín, M. Villatoro, M. A. Delgadillo, E. Mendoza, I. Espejel, P. MorenoCasasola, M. L. Martínez, D. Infante-Mata. 2013. Manejo de ecosistemas de dunas costeras, criterios ecológicos y estrategias. Secretaria de Medio Ambiente y Recursos Naturales. México, D.F., México. 97 pp.

Peinado, M., F. Alcaraz, J. L. Aguirre y J. Álvarez. 1994. Vegetation formations and associations of the zonobiomes along the North American Pacific coast. Vegetatio 114(2): 123-135.

Peinado, M., F. M. Ocana-Peinado, J. L. Aguirre, J. Delgadillo, M. A. Macias y G. Díaz-Santiago. 2011. A phytosociological and phytogeographical survey of the coastal vegetation of western North America: beach and dune vegetation from Baja California to Alaska. Applied Vegetation Science 14(4): 464-484. DOI: http://dx.doi.org/10.1111/ j.1654-109X.2011.01134.X

SEMARNAT. 2010. Norma Oficial Mexicana NOM-059-SEMARNAT-2010. Protección ambiental-Especies nativas de México de flora y fauna silvestres-Categorías de riesgo y especificaciones para su inclusión, exclusión o cambioLista de especies en riesgo. Secretaria de Medio Ambiente y Recursos Naturales. Diario Oficial de la Federación. México, Cd. Mx., México. http://dof.gob.mx/nota_detalle.php? codigo $=5173091 \&$ fecha $=30 / 12 / 2010$ (consultado julio de 2015).

Seingier, G., I. Espejel y J. L. F. Almada. 2009. Cobertura vegetal y marginación en la costa mexicana. Investigación ambiental: Ciencia y política pública 1: 54-69.
StatSoft, Inc. 2007. STATISTICA (data analysis software system), version 8.0. ww.statsoft.com.

Steinmann, V. W. 2002. Diversidad y endemismo de la familia Euphorbiaceae en México. Acta Botanica Mexicana 61: 61-93. DOI: http://dx.doi.org/10.21829/abm61.2002.909

Torres, W., M. Méndez, A. Dorantes y R. Durán. 2010. Estructura, composición y diversidad del matorral de duna costera en el litoral Yucateco. Boletín de la Sociedad Botánica de México 86: 37-51.

TROPICOS. 2016. Tropicos.org. Missouri Botanical Garden. http://www.tropicos.org (consultado 2015 y 2016).

Vanderplank, S. E. 2011. The Flora of Greater San Quintin, Baja California, Mexico (2005-2010). Aliso 29(2): 65103. DOI: http://dx.doi.org/10.5642/aliso.20112902.02

Vega-Aviña R. y P. Ramos-Rivera. 2015. Catálogo y base de datos preliminar de la flora de Sinaloa. Comisión Nacional para el Conocimiento y Uso de la Biodiversidad. DOI: http://doi.org/10.15468/rdm1 oa

Villaseñor, J. L. y F. J. Espinosa. 1998. Catálogo de malezas de México. Universidad Nacional Autónoma de México. Consejo Nacional Consultivo Fitosanitario. Fondo de Cultura Económica. México, D.F., México. 449 pp.

Villaseñor, J. L. 2004. Los géneros de plantas vasculares de la flora de México. Boletín de la Sociedad Botánica de México 75: 105-135.

Villaseñor, J. L. y E. Ortiz. 2014. Biodiversidad de las plantas con flores (División Magnoliophyta) en México. Revista Mexicana de Biodiversidad 85: 134-142. DOI: http:// dx.doi.org/10.7550/rmb.31987 
Apéndice. Listado florístico de playas y dunas costeras de México. El asterisco se refiere a las especies que los autores reconocen como mayoritariamente de dunas.

PTERIDOPHYTA

Blechnaceae

Blechnum serrulatum Rich.

Lygodiaceae

Lygodium venustum Sw.

Polypodiaceae

Polypodium palmeri Maxon

Pteridaceae

Vittaria lineata (L.) Sm.

Schizaeaceae

Schizaea elegans (Vahl) Sw.

GIMNOSPERMAS

Zamiaceae

Zamia furfuracea Aiton

Zamia loddigesii Miq.

MAGNOLIOPHYTA (ANGIOSPERMAS)

\section{MAGNOLIDES}

Annonaceae

Annona glabra L.

Annona reticulata $\mathrm{L}$.

Cymbopetalum baillonii R.E. Fr.

Desmopsis galeottiana (Baill.) Saff.

Desmopsis trunciflora (Schltdl. \& Cham.) G.E. Schatz

Mosannona depressa (Baill.) Chatrou

Sapranthus campechianus (Kunth) Standl.

Sapranthus microcarpus (Donn. Sm.) R.E. Fr.

Aristolochiaceae

Aristolochia grandiflora Sw.

Aristolochia ovalifolia Duch.

Aristolochia pentandra Jacq.

Cabombaceae

Cabomba aquatica Aubl.
Hernandiaceae

Gyrocarpus americanus Jacq.

Gyrocarpus jatrophifolius Domin

Lauraceae

Cassytha filiformis L.

Nectandra coriacea (Sw.) Griseb.

Nectandra salicifolia (Kunth) Nees

Ocotea uxpanapana T. Wendt \& van der Werff

Nymphaeaceae

Nymphaea conardii Wiersema

Nymphaea pulchella DC.

Piperaceae

Peperomia angustata Kunth

Peperomia obtusifolia (L.) A. Dietr.

Piper aduncum L.

Piper amalago L.

Piper dilatatum Rich.

Piper nudum C. DC.

Piper pseudolindenii C. DC.

Saururaceae

Anemopsis californica Nutt. ex Hook. \& Arn.

\section{MONOCOTILEDÓNEAS}

Alismataceae

Sagittaria lancifolia L.

Amaryllidaceae

Crinum americanum $\mathrm{L}$.

Hymenocallis americana (Mill.) Roem.

Hymenocallis littoralis (Jacq.) Salisb.

Pancratium littorale Jacq.

Araceae

Anthurium pentaphyllum (Aubl.) G. Don

Anthurium schlechtendalii Kunth

Monstera acuminata K. Koch

Philodendron jacquinii Schott

Philodendron radiatum Schott

Philodendron sagittifolium Liebm.

Pistia stratiotes $\mathrm{L}$. 
Apéndice. Continuación.

Spathiphyllum cochlearispathum (Liebm.) Engl.

Syngonium angustatum Schott

Syngonium podophyllum Schott

Arecaceae

Acoelorrhaphe wrightii (Griseb. \& H. Wendl. ex Griseb.) H. Wendl. ex Becc.

Astrocaryum mexicanum Liebm. ex Mart.

Attalea butyracea (Mutis ex L. f.) Wess. Boer

Chamaedorea elegans Mart.

Chamaedorea seifrizii Burret

Coccothrinax argentata (Jacq.) L.H. Bailey

Cocos nucifera L.*

Pseudophoenix sargentii H. Wendl. ex Sarg.

Sabal mexicana Mart.

Sabal yapa C. Wright ex Becc.

Scheelea liebmannii Becc.

Thrinax radiata Lodd. ex Schult. \& Schult. f.*

Asparagaceae

Agave angustifolia Haw.

Agave colimana Gentry

Agave datylio F.A.C. Weber

Agave felgeri Gentry

Agave margaritae Brandegee

Dichelostemma capitatum (Benth.) Alph. Wood

Hesperocallis undulata A. Gray

Triteleiopsis palmeri (S. Watson) Hoover

Bromeliaceae

Aechmea bracteata (Sw.) Griseb.

Bromelia pinguin $\mathrm{L}$.

Pitcairnia recurvata (Scheidw.) K. Koch

Tillandsia balbisiana Schult. f.

Tillandsia bulbosa Hook.

Tillandsia dasyliriifolia Baker

Tillandsia exserta Fernald

Tillandsia ionantha Planch.

Tillandsia paucifolia Baker

Tillandsia streptophylla Scheidw. ex C. Morren

Tillandsia utriculata L.

Tillandsia variabilis Schltdl.

Commelinaceae

Commelina diffusa Burm. $\mathrm{f}$.

Commelina erecta $\mathrm{L}$.
Murdannia nudiflora (L.) Brenan

Tradescantia spathacea $\mathrm{Sw}$.

Costaceae

Costus pulverulentus C. Presl

Cyperaceae

Bolboschoenus maritimus (L.) Palla

Cladium jamaicense Crantz

Cyperus amabilis Vahl

Cyperus articulatus L.

Cyperus canus J. Presl \& C. Pres1

Cyperus compressus L.

Cyperus cuspidatus Kunth

Cyperus dentoniae G.C. Tucker

Cyperus dioicus I.M. Johnst.

Cyperus elegans L.

Cyperus eragrostis Lam.

Cyperus erythrorhizos Muhl.

Cyperus esculentus L.

Cyperus giganteus Vahl

Cyperus hermaphroditus (Jacq.) Standl.

Cyperus humilis Kunth

Cyperus imbricatus Retz.

Cyperus iria L.

Cyperus laevigatus L.

Cyperus laxus Lam.

Cyperus ligularis $\mathrm{L}$.

Cyperus ochraceus Vahl

Cyperus odoratus $\mathrm{L}$.

Cyperus perennis (M.E. Jones) O’Neill

Cyperus planifolius Rich.

Cyperus polystachyos (Rottb.) P. Beauv.

Cyperus retroflexus Buckley

Cyperus rotundus $\mathrm{L}$.

Cyperus squarrosus L.

Cyperus surinamensis Rottb.

Cyperus tenuis Sw.

Cyperus thyrsiflorus Jungh.

Eleocharis acutangula (Roxb.) Schult.

Eleocharis cellulosa Torr.

Eleocharis elegans (Kunth) Roem. \& Schult.

Eleocharis filiculmis Kunth

Eleocharis geniculata (L.) Roem. \& Schult.

Eleocharis mutata (L.) Roem. \& Schult.

Fimbristylis caroliniana (Lam.) Fernald 
Apéndice. Continuación.

Fimbristylis cymosa R. Br.*

Fimbristylis dichotoma (L.) Vahl

Fimbristylis spadicea (L.) Vahl*

Fuirena simplex Vahl

Lipocarpha micrantha (Vahl) G.C. Tucker

Rhynchospora colorata (L.) H. Pfeiff.

Rhynchospora floridensis (Britton) H. Pfeiff.

Rhynchospora holoschoenoides (Rich.) Herter

Rhynchospora nervosa (Vahl) Boeck.

Rhynchospora rugosa (Vahl) Gale

Rhynchospora velutina (Kunth) Boeck.

Schoenoplectus americanus (Pers.) Volkart ex Schinz \& R. Keller.

Schoenoplectus californicus (C.A. Mey.) Soják

Schoenoplectus pungens (Vahl) Palla

Scleria eggersiana Boeck.

Scleria melaleuca Rchb. ex Schltdl. \& Cham.

Dioscoreaceae

Dioscorea pilosiuscula Bertero ex Spreng.

Heliconiaceae

Heliconia latispatha Benth.

Iridaceae

Alophia veracruzana Goldblatt \& T.M. Howard

Eleutherine latifolia (Standl. \& L.O. Williams) Ravenna

Sisyrinchium biforme E.P. Bicknell

Juncaceae

Juncus acutus L.

Juncus bufonius L.

Marantaceae

Thalia geniculata $\mathrm{L}$.

Orchidaceae

Cyrtopodium macrobulbon (La Llave \& Lex.) G.A. Romero-

Gonzalez \& Carnevali

Cyrtopodium punctatum (L.) Lindl.

Encyclia adenocarpon (La Llave \& Lex.) Schltr.

Encyclia nematocaulon (A. Rich.) Acuña

Epidendrum difforme Jacq.

Habenaria bractescens Lindl.

Habenaria quinqueseta (Michx.) Eaton

Habenaria repens Nutt.

Heterotaxis sessilis (Sw.) F. Barros
Javieria grandiflora (Lindl.) Archila, Chiron \& Szlach.

Javieria nodosa (L.) Archila, Chiron \& Szlach.

Lophiaris lurida (Lindl.) Braem

Oeceoclades maculata (Lindl.) Lindl.

Schomburgkia tibicinis (Bateman) Bateman

Spiranthes costaricensis Rchb. f.

Trichocentrum lindenii (Brongn.) M.W. Chase \& N.H. Williams

Poaceae

Andropogon bicornis L.

Andropogon gerardi Vitman

Andropogon gyrans Ashe

Andropogon scoparius Michx.*

Andropogon selloanus (Hack.) Hack.

Andropogon virginicus $\mathrm{L}$.

Aristida adscensionis L.

Aristida appressa Vasey

Aristida arizonica Vasey

Aristida californica Thurb.*

Aristida divaricata Humb. \& Bonpl. ex Willd.

Aristida floridana (Chapm.) Vasey

Aristida jorullensis Kunth

Aristida laxa Cav.

Aristida pansa Wooton \& Standl.

Aristida purpurea Nutt.

Aristida purpusiana Hitchc.

Aristida tenuifolia Hitchc.

Aristida ternipes Cav.

Arundo donax L.

Avena barbata Pott ex Link

Axonopus centralis Chase

Axonopus flexuosus (Peter) C.E. Hubb.

Bothriochloa barbinodis (Lag.) Herter

Bothriochloa pertusa (L.) A. Camus

Bouteloua annua Swallen

Bouteloua aristidoides (Kunth) Griseb.

Bouteloua barbata Lag.

Bouteloua curtipendula (Michx.) Torr.

Bouteloua erecta (Vasey \& Hack.) Columbus

Bouteloua media (E. Fourn.) Gould \& Kapadia

Bouteloua parryi (E. Fourn.) Griffiths

Bouteloua reflexa Swallen

Bouteloua repens (Kunth) Scribn. \& Merr.*

Bouteloua triaena (Trin. ex Spreng.) Scribn.

Bouteloua trifida Thurb. ex S. Watson

Bouteloua williamsii Swallen 
Apéndice. Continuación.

Brachiaria fasciculata (Sw.) Parodi

Brachiaria mollis (Sw.) Parodi

Bromus catharticus Vahl

Bromus diandrus Roth

Bromus hordeaceus L.

Cenchrus brownii Roem. \& Schult.

Cenchrus ciliaris L.

Cenchrus echinatus L.

Cenchrus incertus M.A. Curtis*

Cenchrus palmeri Vasey*

Cenchrus pilosus Kunth

Chloris barbata $\mathrm{Sw}$.

Chloris crinita Lag.

Chloris gayana Kunth

Chloris virgata $\mathrm{Sw}$.

Chondrosum barbatum (Lag.) Clayton

Cynodon dactylon (L.) Pers.

Cynodon plectostachyus (K. Schum.) Pilg.

Dactyloctenium aegyptium (L.) Willd.

Dichanthium aristatum (Poir.) C.E. Hubb.

Digitaria bicornis (Lam.) Roem. \& Schult.

Digitaria ciliaris (Retz.) Koeler

Digitaria horizontalis Willd.

Dinebra viscida (Scribn.) P.M. Peterson \& N. Snow

Diplachne fusca (L.) P. Beauv. ex Roem. \& Schult.

Disakisperma dubium (Kunth) P.M. Peterson \& N. Snow

Distichlis bajaensis H.L. Bell

Distichlis littoralis (Engelm.) H.L. Bell \& J.T. Columbus

Distichlis palmeri (Vasey) Fassett

Distichlis spicata (L.) Greene*

Echinochloa colona (L.) Link

Eleusine indica (L.) Gaertn.

Enneapogon desvauxii P. Beauv.

Eragrostis cilianensis (All.) Vignolo ex Janch.

Eragrostis ciliaris (L.) R. Br.

Eragrostis elliottii S. Watson

Eragrostis intermedia Hitchc.

Eragrostis pectinacea (Michx.) Nees

Eragrostis pilosa (L.) P. Beauv.

Eragrostis prolifera (Sw.) Steud.*

Eragrostis reptans (Michx.) Nees

Eragrostis scaligera Salzm. ex Steud.

Eragrostis secundiflora subsp. oxylepis (Torr.) S.D. Koch

Eragrostis tenella (L.) P. Beauv. ex Roem. \& Schult.

Eragrostis viscosa (Retz.) Trin.*

Eriochloa acuminata (J. Presl) Kunth
Eriochloa aristata Vasey

Eustachys petraea (Sw.) Desv.*

Festuca octoflora Walter

Hemarthria altissima (Poir.) Stapf \& C.E. Hubb.

Heteropogon contortus (L.) P. Beauv. ex Roem. \& Schult.

Hilaria rigida (Thurb.) Benth. ex Scribn.

Hildaea pallens (Sw.) C. Silva \& R.P. Oliveira

Homolepis aturensis (Kunth) Chase

Hordeum murinum L.

Hordeum vulgare $\mathrm{L}$.

Hymenachne amplexicaulis (Rudge) Nees

Hyparrhenia rufa (Nees) Stapf

Jouvea pilosa (J. Presl) Scribn.*

Lamarckia aurea (L.) Moench

Lasiacis ligulata Hitchc. \& Chase

Lasiacis rugelii (Griseb.) Hitchc.

Lasiacis ruscifolia (Kunth) Hitchc.

Leptochloa filiformis (Pers.) P. Beauv.

Lithachne pauciflora (Sw.) P. Beauv.

Lolium perenne L.

Monerma cylindrica (Willd.) Coss. \& Durieu

Muhlenbergia gigantea (E. Fourn.) Hitchc.

Muhlenbergia microsperma (DC.) Kunth

Oedochloa lanceolata (Scribn. \& J.G. Sm.) C. Silva \& R.P. Oliveira

Oplismenus hirtellus (L.) P. Beauv.

Panicum alatum Zuloaga \& Morrone*

Panicum amarum Elliott*

Panicum chapmanii Vasey

Panicum gouinii E. Fourn.

Panicum hallii Vasey

Panicum hirsutum Sw.

Panicum hirticaule J. Presl

Panicum laxiflorum Lam.

Panicum laxum Sw.

Panicum maximum Jacq.

Panicum pilosum $\mathrm{Sw}$.

Panicum virgatum L.

Pappophorum pappiferum (Lam.) Kuntze

Paratheria prostrata Griseb.

Paspalidium chapmanii (Vasey) R.W. Pohl

Paspalidium geminatum (Forssk.) Stapf

Paspalum blodgettii Chapm.

Paspalum botterii (E. Fourn.) Chase

Paspalum candidum (Humb. \& Bonpl. ex Flüggé) Kunth

Paspalum conjugatum P.J. Bergius

Paspalum distichum $\mathrm{L}$. 
Apéndice. Continuación.

Paspalum millegrana Schrad.

Paspalum monostachyum Vasey

Paspalum virgatum $\mathrm{L}$.

Phalaris caroliniana Walter

Phalaris minor Retz.

Pharus lappulaceus Aubl.

Phragmites communis Trin.

Polypogon monspeliensis (L.) Desf.

Rhynchelytrum repens (Willd.) C.E. Hubb.

Schizachyrium condensatum (Kunth) Nees

Schizachyrium muelleri Nash

Setaria macrostachya Kunth

Setaria palmeri Henrard

Setaria parviflora (Poir.) Kerguélen

Setariopsis auriculata (E. Fourn.) Scribn.

Sorghum halepense (L.) Pers.

Spartina spartinae (Trin.) Merr. ex Hitchc.

Sporobolus airoides (Torr.) Torr.

Sporobolus contractus Hitchc.

Sporobolus cryptandrus (Torr.) A. Gray

Sporobolus domingensis (Trin.) Kunth

Sporobolus foliosus (Trin.) P.M. Peterson \& Saarela

Sporobolus indicus (L.) R. Br.*

Sporobolus jacquemontii Kunth

Sporobolus pumilus (Roth) P.M. Peterson \& Saarela

Sporobolus virginicus (L.) Kunth*

Sporobolus wrightii Munro ex Scribn.

Stipa mucronata Kunth

Tragus berteronianus Schult.

Trichachne californica (Benth.) Chase

Triplasis purpurea (Walter) Chapm.

Uniola paniculata L.*

Uniola pittieri Hack.

Urochloa arizonica (Scribn. \& Merr.) Morrone \& Zuloaga

Urochloa distachya (L.) T.Q. Nguyen

Urochloa mutica (Forssk.) T.Q. Nguyen

Urochloa plantaginea (Link) R.D. Webster

Urochloa ramosa (L.) T.Q. Nguyen

Vulpia octoflora (Walter) Rydb.

Pontederiaceae

Pontederia sagittata C. Presl

Ruppiaceae

Ruppia maritima L.
Smilacaceae

Smilax aristolochiifolia Mill.

Smilax domingensis Willd.

Smilax mollis Humb. \& Bonpl. ex Willd.

Typhaceae

Typha domingensis Pers.

Zingiberaceae

Renealmia mexicana Klotzsch ex Petersen

\section{EUDICOTILEDÓNEAS}

Acanthaceae

Aphelandra scabra (Vahl) Sm.

Avicennia germinans (L.) L.

Blechum brownei Juss.

Bravaisia berlandieriana (Nees) T.F. Daniel

Bravaisia integerrima (Spreng.) Standl.

Dicliptera acuminata (Ruiz \& Pav.) Juss.

Dicliptera sexangularis (L.) Juss.

Elytraria imbricata (Vahl) Pers.

Justicia californica (Benth.) D.N. Gibson

Justicia candicans (Nees) L.D. Benson

Justicia carthagenensis Jacq.

Justicia fulvicoma Schltdl. \& Cham.

Justicia salma-margaritae Acosta Cast.

Odontonema callistachyum (Schltdl. \& Cham.) Kuntze

Ruellia californica (Rose) I.M. Johnst.

Ruellia ciliatiflora Hook.

Ruellia foetida Willd.

Stenandrium nanum (Standl.) T.F. Daniel

Achatocarpaceae

Achatocarpus gracilis $\mathrm{H}$. Walter

Achatocarpus nigricans Triana

Achatocarpus oaxacanus Standl.

Phaulothamnus spinescens A. Gray

\section{Aizoaceae}

Carpobrotus chilensis (Molina) N.E. Br.

Mesembryanthemum crystallinum L.

Mesembryanthemum nodiflorum $\mathrm{L}$.

Sesuvium maritimum (Walter) Britton, Sterns \& Poggenb.

Sesuvium verrucosum Raf.*

Trianthema portulacastrum $\mathrm{L}$. 
Apéndice. Continuación.

Amaranthaceae

Achyranthes aspera L.

Allenrolfea occidentalis (S. Watson) Kuntze

Alternanthera brasiliana (L.) Kuntze

Alternanthera flavescens Kunth

Alternanthera gracilis (M. Martens \& Galeotti) Loes.

Alternanthera lanceolata (Benth.) Schinz

Alternanthera stellata Uline \& W.L. Bray

Amaranthus albus L.

Amaranthus deflexus L.

Amaranthus dubius Mart. ex Thell.

Amaranthus fimbriatus (Torr.) Benth. ex S. Watson

Amaranthus greggii S. Watson*

Amaranthus hybridus L.

Amaranthus lepturus S.F. Blake

Amaranthus palmeri S. Watson

Amaranthus retroflexus L.

Amaranthus scariosus Benth.

Amaranthus spinosus L.

Amaranthus watsonii Standl.

Aphanisma blitoides Nutt. ex Moq.

Arthrocnemum indicum (Willd.) Moq.

Arthrocnemum subterminale (Parish) Standl.

Atriplex arenaria Nutt.

Atriplex barclayana (Benth.) D. Dietr.

Atriplex canescens (Pursh) Nutt.

Atriplex coulteri (Moq.) D. Dietr.

Atriplex erosa Brueckner \& Verdoorn

Atriplex frankenioides Moran

Atriplex hymenelytra (Torr.) S. Watson

Atriplex julacea S. Watson

Atriplex leucophylla (Moq.) D. Dietr.

Atriplex lindleyi Moq.

Atriplex linearis S. Watson

Atriplex magdalenae Brandegee

Atriplex pacifica A. Nelson

Atriplex polycarpa (Torr.) S. Watson

Atriplex prostrata Boucher ex DC.

Atriplex semibaccata R. Br.

Atriplex serenana A. Nelson ex Abrams

Atriplex suberecta I. Verd.

Atriplex watsonii A. Nelson ex Abrams

Bassia hyssopifolia (Pall.) Kuntze

Beta vulgaris L.

Blutaparon vermiculare (L.) Mears*

Celosia floribunda A. Gray
Celosia nitida Vahl

Chamissoa altissima (Jacq.) Kunth

Chenopodium californicum (S.Watson) S.Watson

Chenopodium flabellifolium Standl.

Chenopodium murale L.

Extriplex californica (Moq.) E.H. Zacharias

Froelichia interrupta (L.) Moq.

Froelichia xantusii R.A. McCauley

Gomphrena decumbens Jacq.*

Gomphrena filaginoides M. Martens \& Galeotti

Gomphrena serrata L.

Gomphrena sonorae Torr.

Gossypianthus lanuginosus (Poir.) Moq.

Iresine alternifolia $\mathrm{S}$. Watson

Iresine angustifolia Euphrasén

Iresine diffusa Humb. \& Bonpl. ex Willd.

Iresine flavescens Humb. \& Bonpl. ex Willd.

Iresine heterophylla Standl.

Iresine paniculata Poir.

Lagrezia monosperma (Rose) Standl.

Philoxerus vermicularis (L.) R. Br. ex Sm.

Salicornia bigelovii Torr.

Salicornia depressa Standl.

Salicornia virginica L.

Salsola kali L.

Sarcocornia pacifica (Standl.) A.J. Scott

Suaeda californica $\mathrm{S}$. Watson

Suaeda esteroa Ferren \& S.A. Whitmore

Suaeda linearis (Elliott) Moq.

Suaeda nigra J.F. Macbr.

Suaeda plumosa Aellen

Suaeda tampicensis (Standl.) Standl.

Suaeda taxifolia (Standl.) Standl.

Tidestromia lanuginosa (Nutt.) Standl.

Anacardiaceae

Amphipterygium adstringens (Schltdl.) Standl.

Astronium graveolens Jacq.

Comocladia engleriana Loes.

Cyrtocarpa edulis (Brandegee) Standl.

Cyrtocarpa procera Kunth

Mangifera indica $\mathrm{L}$.

Metopium brownei (Jacq.) Urb.

Pachycormus discolor (Benth.) Coville

Pseudosmodingium perniciosum (Kunth) Engl.

Rhus aromatica Aiton 
Apéndice. Continuación.

Rhus integrifolia (Nutt.) Benth. \& Hook. f. ex W.H. Brewer \& S. Watson

Spondias mombin L.

Spondias purpurea $\mathrm{L}$.

Apiaceae

Apium graveolens $\mathrm{L}$.

Bowlesia incana Ruiz \& Pav.

Centella asiatica (L.) Urb.

Eryngium foetidum L.

Eryngium nasturtiifolium Juss. ex F. Delaroche Spermolepis echinata (Nutt. ex DC.) A. Heller

Apocynaceae

Asclepias albicans S. Watson

Asclepias curassavica L.

Asclepias oenotheroides Schltdl. \& Cham.

Asclepias subulata Decne.

Aspidosperma megalocarpon Müll. Arg.

Blepharodon mucronatum (Schltdl.) Decne.

Calotropis procera (Aiton) W.T. Aiton

Cameraria latifolia $\mathrm{L}$.

Catharanthus roseus (L.) G. Don

Cryptostegia madagascariensis Bojer ex Decne.

Cynanchum foetidum (Cav.) Kunth

Echites umbellatus Jacq.

Echites yucatanensis Millsp. ex Standl.

Forsteronia myriantha Donn. Sm.

Funastrum arenarium (Decne. ex Benth.) Liede

Funastrum clausum (Jacq.) Schltr.

Funastrum hartwegii (Vail) Schltr.

Gonolobus barbatus Kunth

Mandevilla subsagittata (Ruiz \& Pav.) Woodson

Marsdenia callosa Juárez-Jaimes \& W.D. Stevens

Marsdenia edulis S. Watson

Marsdenia propinqua Hemsl.

Matelea aenea (Woodson) W.D. Stevens

Matelea belizensis (Lundell \& Standl.) Woodson

Matelea cordifolia (A. Gray) Woodson

Matelea fruticosa (Brandegee) Woodson

Matelea pringlei (A. Gray) Woodson

Matelea umbellata (Brandegee) Woodson

Mesechites trifidus (Jacq.) Müll. Arg.

Metastelma arizonicum A. Gray

Metastelma barbigerum Scheele

Metastelma californicum Benth.
Metastelma palmeri S. Watson

Metastelma pringlei A. Gray

Metastelma schlechtendalii Decne.

Nerium oleander L.

Pentalinon andrieuxii (Müll. Arg.) B.F. Hansen \& Wunderlin

Pinochia peninsularis (Woodson) M.E. Endress \& B.F. Hansen

Plumeria obtusa L.

Plumeria rubra $\mathrm{L}$.

Prestonia mexicana A. DC.

Prestonia portobellensis (Beurl.) Woodson

Rauvolfia ligustrina Willd. ex Roem. \& Schult.

Rauvolfia tetraphylla $\mathrm{L}$.

Rhabdadenia biflora (Jacq.) Müll. Arg.*

Seutera palmeri (S. Watson) Fishbein \& W.D. Stevens

Stemmadenia pubescens Benth.

Tabernaemontana alba Mill.

Tabernaemontana amygdalifolia Jacq.

Tabernaemontana donnell-smithii Rose

Tabernaemontana litoralis Kunth

Tabernaemontana odontadeniiflora A.O. Simões \& M.E. Endress

Thevetia ahouai (L.) A. DC.

Thevetia gaumeri Hemsl.

Thevetia ovata (Cav.) A. DC.

Thevetia peruviana $\mathrm{K}$. Schum.

Vallesia antillana Woodson*

Vallesia laciniata Brandegee

Araliaceae

Dendropanax arboreus (L.) Decne. \& Planch.

Hydrocotyle bonariensis Lam.

Hydrocotyle verticillata Thunb.

Asteraceae

Adenophyllum aurantium (L.) Strother

Adenophyllum speciosum (A. Gray) Strother

Adenothamnus validus (Brandegee) D.D. Keck

Ageratum conyzoides L.

Ageratum maritimum Kunth

Aldama fruticosa (Brandegee) E. E. Schill. \& Panero

Alvordia fruticosa Brandegee

Amauria rotundifolia Benth.

Amblyopappus pusillus Hook. \& Arn.

Ambrosia artemisiifolia $\mathrm{L}$.

Ambrosia camphorata (Greene) W.W. Payne

Ambrosia chamissonis (Less.) Greene

Ambrosia chenopodiifolia (Benth.) W.W. Payne 
Apéndice. Continuación.

Ambrosia confertiflora DC.

Ambrosia cordifolia (A. Gray) W.W. Payne

Ambrosia cumanensis Kunth

Ambrosia deltoidea (Torr.) W.W. Payne

Ambrosia divaricata (Brandegee) W.W. Payne

Ambrosia dumosa (A. Gray) W.W. Payne

Ambrosia hispida Pursh*

Ambrosia magdalenae (Benth.) W.W. Payne

Ambrosia monogyra (Torr. \& A. Gray) Strother \& B.G. Baldwin

Ambrosia psilostachya DC.

Ambrosia salsola (Torr. \& A. Gray) Strother \& B.G. Baldwin

Artemisia vulgaris L.

Baccharis dioica Vahl*

Baccharis heterophylla Kunth

Baccharis salicina Torr. \& A. Gray

Baccharis sergiloides A. Gray

Baccharis trinervis Pers.

Bahiopsis chenopodina (Greene) E.E. Schill. \& Panero

Bahiopsis deltoidea (A. Gray) E.E. Schill. \& Panero

Bahiopsis laciniata (A. Gray) E.E. Schill. \& Panero

Bahiopsis lanata Kellogg

Bahiopsis microphylla (Vasey \& Rose) E. E. Schill. \& Panero

Baileya pleniradiata Harv. \& A. Gray

Bajacalia crassifolia (S. Watson) Loockerman, B.L. Turner \& R.K.

Jansen

Bajacalia moranii B.L. Turner

Bajacalia tridentata (Benth.) Loockerman, B.L. Turner \& R.K. Jansen

Baltimora recta $\mathrm{L}$.

Bebbia atriplicifolia (A. Gray) Greene

Bebbia juncea (Benth.) Greene

Bidens anthemoides (DC.) Sherff*

Bidens cabopulmensis León de la Luz*

Bidens odorata Cav.

Bidens pilosa $\mathrm{L}$.

Bidens squarrosa Kunth

Blumea viscosa (Mill.) V.M. Badillo

Boeberastrum anthemidifolia (Benth.) Rydb.

Boeberastrum littoralis (Brandegee) Rydb.

Borrichia arborescens (L.) DC.*

Borrichia frutescens (L.) DC.*

Brickellia californica (Torr. \& A. Gray) A. Gray

Brickellia frutescens A. Gray

Brickellia glabrata (Rose) B.L. Rob.

Calea jamaicensis (L.) L.

Calea urticifolia (Mill.) DC.

Chaenactis carphoclinia A. Gray
Chaenactis glabriuscula DC.

Chaenactis lacera Greene

Chaenactis stevioides Hook. \& Arn.

Chloracantha spinosa (Benth.) G. L. Nesom

Chromolaena lundellii R.M. King \& H. Rob.

Chromolaena odorata (L.) R.M. King \& H. Rob.

Chromolaena sagittata (A. Gray) R.M. King \& H. Rob.

Conoclinium betonicifolium (Mill.) R.M. King \& H. Rob.

Conyza canadensis (L.) Cronquist

Coreocarpus dissectus (Benth.) S.F. Blake

Coreocarpus involutus Greene

Coreocarpus parthenioides Benth.

Coreocarpus sonoranus Sherff

Corethrogyne filaginifolia (Hook. \& Arn.) Nutt.

Cotula coronopifolia $\mathrm{L}$.

Coulterella capitata Vasey \& Rose

Critonia morifolia (Mill.) R.M. King \& H. Rob.

Deinandra fasciculata (DC.) Greene

Dicoria canescens A. Gray*

Dyssodia anthemidifolia Benth.

Eclipta prostrata (L.) L.

Elephantopus mollis Kunth

Emilia fosbergii Nicolson

Emilia sonchifolia (L.) DC.

Encelia californica Nutt.

Encelia conspersa Benth.

Encelia farinosa A. Gray ex Torr.

Encelia halimifolia Cav.*

Encelia laciniata Vasey \& Rose

Encelia palmeri Vasey \& Rose

Encelia ventorum Brandegee*

Epaltes mexicana Less.

Erechtites hieraciifolius (L.) Raf. ex DC.

Ericameria brachylepis (A. Gray) H.M. Hall

Ericameria palmeri (A. Gray) H.M. Hall

Ericameria parishii (Greene) H.M. Hall

Erigeron bonariensis L.

Erigeron canadensis L.

Erigeron longipes DC.

Erigeron procumbens (Houst. ex Mill.) G.L. Nesom

Espejoa mexicana DC.

Eupatorium albicaule Sch. Bip. ex Klatt

Eupatorium betonicifolium Mill.

Eupatorium megaphyllum Baker

Eupatorium odoratum L.

Eupatorium peninsulare Brandegee 
Apéndice. Continuación.

Eupatorium pittieri Klatt

Flaveria linearis Lag.

Flaveria trinervia (Spreng.) C. Mohr

Fleischmannia pycnocephala (Less.) R.M. King \& H. Rob.

Florestina liebmannii Sch. Bip. ex Greenm.

Florestina pedata (Cav.) Cass.

Florestina tripteris DC.

Gaillardia pulchella Foug.

Gutierrezia ramulosa (Greene) M.A. Lane

Gutierrezia sarothrae (Pursh) Britton \& Rusby

Hazardia berberidis (A. Gray) Greene

Hazardia squarrosa (Hook. \& Arn.) Greene

Helianthus annuus L.

Helianthus laciniatus A. Gray

Helianthus niveus (Benth.) Brandegee*

Helianthus petiolaris Nutt.

Heliopsis anomala (M.E. Jones) B.L. Turner

Heliopsis parvifolia A. Gray

Heterosperma xanti A. Gray

Heterotheca grandiflora Nutt.

Heterotheca inuloides Cass.

Heterotheca sessiliflora (Nutt.) Shinners

Heterotheca subaxillaris (Lam.) Britton \& Rusby

Hofmeisteria crassifolia S. Watson

Hofmeisteria fasciculata (Benth.) Walp.

Isocoma acradenia (Greene) Greene

Isocoma menziesii (Hook. \& Arn.) G.L. Nesom

Iva angustifolia Nutt. ex DC.

Iva asperifolia Less.*

Jaumea carnosa (Less.) A. Gray

Jefea lantanifolia (S. Schauer) Strother

Lasthenia coronaria (Nutt.) Ornduff

Lasthenia gracilis (DC.) Greene

Launaea intybacea (Jacq.) Beauverd

Leucosyris crispa (Brandegee) Pruski \& R.L. Hartm.

Machaeranthera incisifolia (I.M. Johnst.) G.L. Nesom

Machaeranthera phyllocephala (DC.) Shinners

Malacothrix glabrata (A. Gray ex D.C. Eaton) A. Gray*

Malacothrix xanti A. Gray

Malperia tenuis S. Watson

Matricaria discoidea DC.

Matricaria matricarioides ((Less.) Porter ex Britton)

Melampodium americanum $\mathrm{L}$.

Melampodium divaricatum (Rich.) DC.

Melampodium gracile Less.

Melampodium montanum Benth.
Melanthera aspera (Jacq.) Steud. ex Small

Melanthera nivea (L.) Small

Mikania cordifolia (L. f.) Willd.

Mikania micrantha Kunth

Mikania microptera DC.

Milleria quinqueflora $\mathrm{L}$.

Monoptilon bellioides (A. Gray) H.M. Hall

Neurolaena lobata (L.) Cass.

Nicolletia trifida Rydb.

Packera plattensis (Nutt.) W.A. Weber \& Á. Löve

Palafoxia arida B.L. Turner \& M.I. Morris*

Palafoxia lindenii A. Gray*

Palafoxia linearis (Cav.) Lag.*

Palafoxia rosea (Bush) Cory

Palafoxia texana DC.

Parthenice mollis A. Gray

Parthenium fruticosum Less.

Parthenium hysterophorus L.

Pectis coulteri Harv. \& A. Gray

Pectis exserta McVaugh

Pectis multiflosculosa (DC) Sch. Bip.

Pectis multiseta Benth.

Pectis papposa Harv. \& A. Gray

Pectis prostrata Cav.

Pectis saturejoides (Mill.) Sch. Bip.*

Pectis uniaristata DC.

Pectis vollmeri Wiggins

Perityle aurea Rose

Perityle californica Benth.

Perityle crassifolia Brandegee

Perityle cuneata Brandegee

Perityle emoryi Torr.

Perityle leptoglossa Harv. \& A. Gray

Perityle microglossa Benth.

Perityle socorrosensis Rose

Peucephyllum schottii A. Gray

Pleurocoronis laphamioides (Rose) R.M. King \& H. Rob.

Pleurocoronis pluriseta (A. Gray) R.M. King \& H. Rob.

Pluchea baccharis (Mill.) Pruski

Pluchea carolinensis (Jacq.) G. Don

Pluchea parvifolia (A. Gray) R.K. Godfrey

Pluchea salicifolia S.F. Blake

Porophyllum gracile Benth.

Porophyllum maritimum Brandegee*

Porophyllum pausodynum B.L. Rob. \& Greenm.

Porophyllum punctatum (Mill.) S.F. Blake 
Apéndice. Continuación.

Pseudognaphalium californicum (DC.) Anderb.

Pseudognaphalium stramineum (Kunth) Anderb.

Pseudogynoxys chenopodioides (Kunth) Cabrera

Rafinesquia californica Nutt.

Rafinesquia neomexicana A. Gray

Rayjacksonia phyllocephala (DC.) R.L. Hartm. \& M.A. Lane

Sanvitalia procumbens Lam.

Sclerocarpus divaricatus (Benth.) Benth. \& Hook. F. ex Hemsl.

Sclerocarpus uniserialis (Hook.) Benth. \& Hook. f. ex Hemsl.

Senecio californicus DC.

Senecio longilobus Benth.

Senecio lyonii A. Gray

Senecio mohavensis A. Gray

Sinclairia discolor Hook. \& Arn.

Solidago sempervirens L.

Solidago velutina DC.

Sonchus oleraceus L.

Sonchus tenerrimus L.

Sphagneticola trilobata (L.) Pruski

Spiracantha cornifolia Kunth

Stebbinsoseris heterocarpa (Nutt.) K.L. Chambers

Stephanomeria diegensis Gottlieb

Stephanomeria exigua Nutt.

Stephanomeria pauciflora (Torr.) A. Nelson

Stephanomeria virgata Benth.

Stylocline gnaphaloides Nutt.

Symphyotrichum subulatum (Michx.) G.L. Nesom

Tagetes linifolia Seaton

Tithonia diversifolia (Hemsl.) A. Gray

Trichoptilium incisum (A. Gray) A. Gray

Tridax procumbens $\mathrm{L}$.

Trixis angustifolia DC.

Trixis californica Kellogg

Trixis inula Crantz

Trixis pterocaulis B.L. Rob. \& Greenm.

Verbesina dissita A. Gray

Verbesina persicifolia DC.

Vernonia cinerea (L.) Less.

Vernonia patens Kunth

Viguiera dentata (Cav.) Spreng.

Viguiera lanata (Kellogg) A. Gray

Wedelia acapulcensis Kunth

Wedelia hispida Kunth

Xanthisma arenarium (Benth.) D.R. Morgan \& R.L. Hartm.

Xanthisma incisifolium (I.M. Johnst.) G.L. Nesom

Xanthisma spinulosum (Pursh) D.R. Morgan \& R.L. Hartm.
Xanthium strumarium L.

Xylothamia diffusa (Benth.) G.L. Nesom

Zexmenia serrata La Llave

Zinnia acerosa (DC.) A. Gray

Zinnia angustifolia Kunth

Zinnia maritima Kunth

Balanophoraceae

Helosis cayanensis (Sw.) Spreng.

Bataceae

Batis maritima L.

Berberidaceae

Berberis claireae Moran

Bignoniaceae

Amphilophium paniculatum (L.) Kunth

Amphitecna apiculata A.H. Gentry

Arrabidaea corallina (Jacq.) Sandwith

Arrabidaea floribunda (Kunth) Loes.

Arrabidaea inaequalis (DC. ex Splitg.) Bureau ex K. Schum.

Arrabidaea podopogon (DC.) A.H. Gentry

Arrabidaea pubescens (L.) A.H. Gentry

Arrabidaea verrucosa (Standl.) A.H. Gentry

Clytostoma binatum (Thunb.) Sandwith

Crescentia cujete L.

Cydista aequinoctialis (L.) Miers

Cydista diversifolia (Kunth) Miers

Distictis laxiflora (DC.) Greenm.

Fridericia dichotoma (Jacq.) L.G. Lohmann

Fridericia pubescens (L.) L.G. Lohmann

Handroanthus ochraceus (Cham.) Mattos

Macfadyena unguis-cati (L.) A.H. Gentry

Paragonia pyramidata (Rich.) Bureau

Pithecoctenium crucigerum (L.) A.H. Gentry

Stizophyllum riparium (Kunth) Sandwith

Tabebuia ochracea (Cham.) Standl.

Tabebuia rosea (Bertol.) DC.

Tanaecium pyramidatum (Rich.) L.G. Lohmann

Tecoma impetiginosa Mart. ex DC.

Tecoma stans (L.) Juss. ex Kunth

Bixaceae

Amoreuxia palmatifida DC.

Bixa orellana L. 
Apéndice. Continuación.

Boraginaceae

Amsinckia inepta J.F. Macbr.

Amsinckia intermedia Fisch. \& C.A. Mey.

Amsinckia menziesii (Lehm.) A. Nelson \& J.F. Macbr.

Amsinckia spectabilis Fisch. \& C.A. Mey.

Bourreria ovata Miers

Bourreria pulchra (Millsp.) Millsp.

Bourreria sonorae S. Watson

Cordia alliodora (Ruiz \& Pav.) Oken

Cordia ambigua Schltdl. \& Cham.

Cordia curassavica (Jacq.) Roem. \& Schult.

Cordia dentata Poir.

Cordia foliosa M. Martens \& Galeotti

Cordia globosa (Jacq.) Kunth

Cordia parvifolia A. DC.

Cordia pringlei B.L. Rob.

Cordia sebestena $\mathrm{L}$.

Cordia sonorae Rose

Cordia stellifera I.M. Johnst.

Cordia stenoclada I.M. Johnst.

Cordia truncatifolia Bartlett

Cryptantha clevelandii Greene

Cryptantha intermedia Greene

Cryptantha maritima (Greene) Greene*

Cryptantha micrantha (Torr.) I.M. Johnst.

Cryptantha patula Greene

Cryptantha pondii Greene

Ehretia anacua (Terán \& Berland.) I.M. Johnst.

Ehretia tinifolia L.

Eucrypta chrysanthemifolia (Benth.) Greene

Heliotropium angiospermum Murray

Heliotropium curassavicum L.

Heliotropium filiforme Lehm.

Heliotropium indicum $\mathrm{L}$.

Heliotropium macrostachyum (DC.) Hemsl.

Heliotropium procumbens Mill.

Johnstonella angelica (I.M. Johnst.) Hasenstab \& M.G. Simpson

Johnstonella angustifolia (Torr.) Hasenstab \& M.G. Simpson

Johnstonella echinosepala (J.F. Macbr.) Hasenstab \& M.G. Simpson

Johnstonella grayi (Vasey \& Rose) Hasenstab \& M.G. Simpson

Johnstonella holoptera (A. Gray) Hasenstab \& M.G. Simpson

Johnstonella racemosa (S. Watson ex A. Gray) Brand

Lennoa madreporoides Lex.

Nama biflora Choisy

Nama demissa A. Gray

Phacelia affinis A. Gray
Phacelia crenulata Torr. ex S. Watson

Phacelia cryptantha Greene

Phacelia distans Benth.

Phacelia ixodes Kellogg

Phacelia pedicellata A. Gray

Phacelia ramosissima Douglas ex Lehm.

Phacelia scariosa Brandegee

Phacelia stellaris Brand

Pholisma arenarium Nutt. ex Hook.*

Pholistoma racemosum (Nutt. ex A. Gray) Constance

Rochefortia lundellii Camp

Tiquilia canescens (A. DC.) A.T. Richardson

Tiquilia cuspidata (I.M. Johnst.) A.T. Richardson

Tiquilia palmeri (A. Gray) A.T. Richardson

Tiquilia plicata (Torr.) A.T. Richardson*

Tournefortia densiflora M. Martens \& Galeotti

Tournefortia glabra L.

Tournefortia gnaphalodes (L.) R. Br. ex Roem. \& Schult.*

Tournefortia hirsutissima $\mathrm{L}$.

Tournefortia mutabilis Vent.

Tournefortia volubilis L.

Varronia spinescens (L.) Borhidi

Brassicaceae

Brassica sisymbrioides Grossh.

Cakile edentula (Bigelow) Hook.*

Cakile geniculata (B.L. Rob.) Millsp.

Cakile lanceolata (Willd.) O.E. Schulz*

Cakile maritima Scop.

Cardamine flexuosa With.

Descurainia sophia (L.) Webb ex Prantl

Dithyrea californica Harv.*

Dithyrea maritima (Davidson) Davidson*

Dryopetalon crenatum (Brandegee) Rollins

Dryopetalon palmeri (S. Watson) O.E. Schulz

Erysimum capitatum (Douglas ex Hook.) Greene

Hirschfeldia incana (L.) Lagr.-Fossat

Lepidium lasiocarpum Nutt. ex Torr. \& A. Gray

Lepidium medium Greene

Lepidium virginicum $\mathrm{L}$.

Lobularia maritima (L.) Desv.

Lyrocarpa coulteri Hook. \& Harv.

Lyrocarpa xanti Brandegee

Raphanus sativus L.

Rorippa teres (Michx.) Stuckey

Sisymbrium irio L. 
Apéndice. Continuación.

Sisymbrium orientale L.

Thysanocarpus curvipes Hook.

Burseraceae

Bursera arborea (Rose) L. Riley

Bursera epinnata (Rose) Engl.

Bursera excelsa (Kunth) Engl.

Bursera fagaroides (Kunth) Engl.

Bursera filicifolia Brandegee

Bursera grandifolia (Schltdl.) Engl.

Bursera graveolens (Kunth) Triana \& Planch.

Bursera hindsiana (Benth.) Engl.

Bursera instabilis McVaugh \& Rzed.

Bursera laurihuertae Rzed. \& Calderón

Bursera linanoe (La Llave) Rzed., Calderón \& Medina

Bursera littoralis León de la Luz \& Pérez Navarro*

Bursera microphylla A. Gray

Bursera schlechtendalii Engl.

Bursera simaruba (L.) Sarg.

Protium copal (Schltdl. \& Cham.) Engl.

Cactaceae

Acanthocereus horridus Britton \& Rose

Acanthocereus subinermis Britton \& Rose

Acanthocereus tetragonus (L.) Hummelinck

Bergerocactus emoryi (Engelm.) Britton \& Rose

Cylindropuntia alcahes (F.A.C. Weber) F.M. Knuth

Cylindropuntia bigelovii (Engelm.) F.M. Knuth

Cylindropuntia cholla (F.A.C. Weber) F.M. Knuth

Cylindropuntia fulgida (Engelm.) F.M. Knuth

Cylindropuntia leptocaulis (DC.) F.M. Knuth

Cylindropuntia prolifera (Engelm.) F.M. Knuth

Cylindropuntia sanfelipensis (Rebman) Rebman

Cylindropuntia santamaria (Baxter) Rebman

Cylindropuntia tesajo (Engelm. ex J.M. Coult.) F.M. Knuth

Echinocereus barthelowanus Britton \& Rose

Echinocereus brandegeei (J.M. Coult.) K. Schum.

Echinocereus maritimus (M.E. Jones) K. Schum.

Echinocereus sciurus (K. Brandegee) Dams

Ferocactus fordii (Orcutt) Britton \& Rose

Ferocactus peninsulae (F.A.C. Weber) Britton \& Rose

Ferocactus townsendianus Britton \& Rose

Ferocactus wislizeni (Engelm.) Britton \& Rose

Grusonia invicta (Brandegee) E.F. Anderson

Hylocereus undatus (Haw.) Britton \& Rose

Lophocereus schottii (Engelm.) Brtitton \& Rose
Mammillaria armillata K. Brandegee

Mammillaria blossfeldiana Boed.

Mammillaria capensis (H.E. Gates) R.T. Craig

Mammillaria dioica K. Brandegee

Mammillaria gaumeri (Britton \& Rose) Orcutt*

Mammillaria goodridgei Scheer

Mammillaria halei Brandegee

Mammillaria hutchisoniana (H.E. Gates) Boed.

Mammillaria mazatlanensis K. Schum.

Mammillaria poselgeri Hildm.

Melocactus curvispinus Pfeiff.

Opuntia auberi Pfeiff.

Opuntia bravoana Baxter

Opuntia dillenii (Ker Gawl.) Haw.

Opuntia excelsa Sánchez-Mej.

Opuntia phaeacantha Engelm.

Opuntia pycnantha Engelm.

Opuntia velutina F.A.C. Weber

Pachycereus pecten-aboriginum (Engelm. ex S. Watson) Britton \& Rose

Pachycereus pringlei (S. Watson) Britton \& Rose

Peniocereus cuixmalensis Sánchez-Mej.

Peniocereus striatus (Brandegee) Buxb.

Pereskia lychnidiflora DC.

Pereskiopsis kellermanii Rose

Pereskiopsis porteri (Brandegee ex F.A.C. Weber) Britton \& Rose

Selenicereus donkelaarii (Salm-Dyck) Britton \& Rose

Stenocereus gummosus (Engelm.) A.C. Gibson \& K.E. Horak

Stenocereus thurberi (Engelm.) Buxb.

Campanulaceae

Lobelia berlandieri A. DC.

Lobelia cardinalis L.

Lobelia xalapensis Kunth

Nemacladus glanduliferus Jeps.

Cannabaceae

Aphananthe monoica (Hemsl.) J.-F. Leroy

Celtis iguanaea (Jacq.) Sarg.

Trema micrantha (L.) Blume

Capparaceae

Atamisquea emarginata Miers ex Hook. \& Arn.

Capparidastrum frondosum (Jacq.) Cornejo \& Iltis

Capparidastrum pachaca (Kunth) Hutch.

Capparis baducca $\mathrm{L}$. 
Apéndice. Continuación.

Capparis flexuosa (L.) L.

Crateva tapia $\mathrm{L}$.

Cynophalla verrucosa (Jacq.) J. Presl

Morisonia americana $\mathrm{L}$.

Quadrella incana (Kunth) Iltis \& Cornejo

Quadrella indica (L.) Iltis \& Cornejo

Quadrella isthmensis (Eichler) Hutch.

\section{Caricaceae}

Carica papaya $\mathrm{L}$.

Caryophyllaceae

Achyronychia cooperi Torr. \& A. Gray

Drymaria arenarioides Humb. \& Bonpl. ex Schult.

Drymaria cordata (L.) Willd. ex Schult.

Drymaria debilis Brandegee

Drymaria holosteoides Benth.

Drymaria viscosa $\mathrm{S}$. Watson

Spergula maritima (All.) Pedersen

Spergularia macrotheca (Hornem. ex Cham. \& Schltdl.) Heynh.

Spergularia villosa (Pers.) Cambess.

\section{Casuarinaceae}

Casuarina equisetifolia L.

\section{Celastraceae}

Crossopetalum densiflorum Lundell

Crossopetalum eucymosum (Loes. \& Pittier) Lundell

Crossopetalum filipes (Sprague) Lundell

Crossopetalum gaumeri (Loes.) Lundell

Crossopetalum parviflorum(Hemsl.) Lundell

Crossopetalum rhacoma Crantz* $^{*}$

Crossopetalum uragoga (Jacq.) Kuntze

Elaeodendron trichotomum (Turcz.) Lundell

Hippocratea celastroides Kunth

Hippocratea excelsa Kunth

Hippocratea volubilis $\mathrm{L}$.

Maytenus phyllanthoides Benth.*

Maytenus schippii Lundell

Microtropis schiedeana Loes.

Salacia elliptica (Mart.) G. Don

Salacia impressifolia (Miers) A.C. Sm.

Schaefferia cuneifolia A. Gray

Schaefferia frutescens Jacq.

Semialarium mexicanum (Miers) Mennega

Wimmeria obtusifolia Standl.
Chrysobalanaceae

Chrysobalanus icaco L.*

Couepia polyandra (Kunth) Rose

Cleomaceae

Arivela viscosa (L.) Raf.

Cleome tenuis $\mathrm{S}$. Watson

Cleoserrata serrata (Jacq.) Iltis

Corynandra viscosa (L.) Cochrane \& Iltis

Gynandropsis gynandra (L.) Briq.

Wislizenia palmeri A. Gray

Clusiaceae

Garcinia intermedia (Pittier) Hammel

Combretaceae

Combretum laxum Jacq.

Conocarpus erectus L.

Laguncularia racemosa (L.) C.F. Gaertn.

Terminalia buceras (L.) C. Wright

Terminalia catappa $\mathrm{L}$.

Connaraceae

Rourea glabra Kunth

Convolvulaceae

Calystegia macrostegia (Greene) Brummitt

Cressa truxillensis Kunth

Cuscuta americana $\mathrm{L}$.

Cuscuta desmouliniana Yunck.

Cuscuta leptantha Engelm.

Cuscuta macrocephala W. Schaffn. ex Yunck.

Cuscuta palustris Yunck.

Cuscuta umbellata Kunth

Cuscuta yucatana Yunck.

Dichondra occidentalis House

Evolvulus alsinoides (L.) L.

Ipomoea barbatisepala A. Gray

Ipomoea batatoides Choisy

Ipomoea carnea Jacq.

Ipomoea cholulensis Kunth

Ipomoea fimbriosepala Choisy

Ipomoea gesnerioides J.A. McDonald

Ipomoea hederifolia L.

Ipomoea imperati (Vahl) Griseb.*

Ipomoea indica (Burm.) Merr. 
Apéndice. Continuación.

Ipomoea jalapa (L.) Pursh

Ipomoea microsepala Benth.

Ipomoea pes-caprae (L.) R. Br.*

Ipomoea purpurea (L.) Roth

Ipomoea quamoclit $\mathrm{L}$.

Ipomoea squamosa Choisy

Ipomoea tricolor Cav.

Ipomoea triloba $\mathrm{L}$.

Ipomoea variabilis (Schltdl. \& Cham.) Choisy

Jacquemontia abutiloides Benth.

Jacquemontia agrestis (Mart. ex Choisy) Meisn.

Jacquemontia eastwoodiana I.M. Johnst.

Jacquemontia havanensis (Jacq.) Urb.*

Jacquemontia oaxacana (Meisn.) Hallier $\mathrm{f}$.

Jacquemontia pentanthos (Jacq.) G. Don

Jacquemontia pringlei A. Gray

Merremia aegyptia (L.) Urb.

Merremia aurea (Kellogg) O’Donell

Merremia dissecta (Jacq.) Hallier f.

Merremia platyphylla (Fernald) O'Donell

Merremia quinquefolia (L.) Hallier $\mathrm{f}$.

Operculina pinnatifida (Kunth) O’Donell

Crassulaceae

Crassula connata (Ruiz \& Pav.) A. Berger

Dudleya acuminata Rose

Dudleya albiflora Rose

Dudleya anthonyi Rose

Dudleya arizonica Rose

Dudleya lanceolata (Nutt.) Britton \& Rose

Dudleya rigidiflora Rose

Echeveria pulverulenta Nutt.

Cucurbitaceae

Citrullus lanatus (Thunb.) Matsum. \& Nakai

Cucumis dipsaceus Ehrenb. ex Spach

Cucumis melo L.

Cucurbita argyrosperma $\mathrm{K}$. Koch

Echinopepon minimus (Kellogg) S. Watson

Ibervillea millspaughii (Cogn.) C. Jeffrey

Ibervillea sonorae (S. Watson) Greene

Luffa aegyptiaca Mill.

Luffa operculata (L.) Cogn.

Melothria pendula L.

Momordica charantia L.

Schizocarpum palmeri Cogn. \& Rose
Tumamoca macdougalii Rose

Vaseyanthus brandegeei (Cogn.) Rose

Vaseyanthus insularis (S. Watson) Rose

Cytinaceae

Bdallophytum americanum (R. Br.) Eichler ex Solms

Dilleniaceae

Curatella americana $\mathrm{L}$.

Doliocarpus dentatus (Aubl.) Standl.

Tetracera volubilis L.

Ebenaceae

Diospyros aequoris Standl.

Diospyros intricata (A. Gray) Standl.

Diospyros juruensis A.C. Sm.

Diospyros nigra (J.F. Gmel.) Perr.

Diospyros salicifolia Humb. \& Bonpl. ex Willd.

Diospyros tetrasperma $\mathrm{Sw}$.

Erythroxylaceae

Erythroxylum areolatum L.

Erythroxylum havanense Jacq.

Erythroxylum macrophyllum Cav.

Erythroxylum rotundifolium Lunan

Euphorbiaceae

Acalypha alopecuroidea Jacq.

Acalypha arvensis Poepp.

Acalypha botteriana Müll. Arg.

Acalypha californica Benth.

Acalypha comonduana Millsp.

Acalypha leptopoda Müll. Arg.

Acalypha microcephala Müll. Arg.

Acalypha monostachya Cav.

Acalypha radians Torr.

Acalypha saxicola Wiggins

Acalypha schiedeana Schltdl.

Acalypha tenuifolia Müll. Arg.

Acalypha umbrosa Brandegee

Alchornea latifolia Sw.

Argythamnia lundellii J.W. Ingram

Astraea lobata(L.) Klotzsch

Caperonia castaneifolia (L.) A. St.-Hil.

Cnidoscolus aconitifolius (Mill.) I.M. Johnst.

Cnidoscolus palmeri (S. Watson) Rose 
Apéndice. Continuación.

Cnidoscolus texanus (Müll. Arg.) Small

Cnidoscolus urens (L.) Arthur

Croton billbergianus Müll. Arg.

Croton californicus Müll. Arg.*

Croton capitatus Michx.

Croton ciliatoglandulifer Ortega

Croton cortesianus Kunth

Croton discolor Willd.

Croton glabellus L.

Croton glandulosus L.

Croton humilis L.

Croton peraeruginosus Croizat

Croton pottsii (Klotzsch) Müll. Arg.

Croton punctatus Jacq.*

Croton reflexifolius Kunth

Croton sonorae Torr.

Croton suberosus Kunth

Croton wigginsii L.C. Wheeler*

Dalechampia scandens L.

Ditaxis brandegeei (Millsp.) Rose \& Standl.

Ditaxis lanceolata (Benth.) Pax \& K. Hoffm.

Ditaxis manzanilloana (Rose) Pax \& K. Hoffm.

Ditaxis neomexicana (Müll. Arg.) A. Heller

Ditaxis serrata (Torr.) A. Heller

Enriquebeltrania crenatifolia (Miranda) Rzed.

Euphorbia abramsiana L.C. Wheeler

Euphorbia albomarginata Torr. \& A. Gray

Euphorbia armourii Millsp.

Euphorbia blodgettii Engelm. ex Hitchc.

Euphorbia bombensis Jacq.

Euphorbia californica Benth.

Euphorbia capitellata Engelm.

Euphorbia cymosa Poir.

Euphorbia dentosa I.M. Johnst.

Euphorbia dioeca Kunth

Euphorbia eriantha Benth.

Euphorbia florida Engelm.

Euphorbia francoana Boiss.

Euphorbia heterophylla L.

Euphorbia hindsiana Benth.

Euphorbia hirta L.

Euphorbia hypericifolia L.

Euphorbia hyssopifolia L.

Euphorbia incerta Brandegee

Euphorbia indivisa (Engelm.) Tidestr.

Euphorbia johnstonii Mayfield
Euphorbia leucophylla Benth.*

Euphorbia lomelii V.W. Steinm.

Euphorbia magdalenae Benth.

Euphorbia marginata Pursh

Euphorbia melanadenia Torr.

Euphorbia mesembryanthemifolia Jacq.

Euphorbia micromera Boiss.

Euphorbia misera Benth.*

Euphorbia parishii Greene

Euphorbia pediculifera Engelm.

Euphorbia peninsularis I.M. Johnst.

Euphorbia petrina S. Watson

Euphorbia polycarpa Benth.

Euphorbia pondii Millsp.

Euphorbia prostrata Aiton

Euphorbia schlechtendalii Boiss.

Euphorbia serpens Kunth

Euphorbia setiloba Engelm.

Euphorbia thymifolia L.

Euphorbia tomentulosa S. Watson

Euphorbia trichotoma Kunth

Euphorbia xanti Engelm. ex Boiss.

Euphorbia xbacensis Millsp.

Garcia nutans Vahl

Gymnanthes lucida Sw.

Hippomane mancinella L.

Hura polyandra Baill.

Jatropha cinerea (Ortega) Müll. Arg.

Jatropha cuneata Wiggins \& Rollins

Jatropha gossypiifolia L.

Jatropha ortegae Standl.

Jatropha sympetala S.F. Blake \& Standl.

Manihot aesculifolia (Kunth) Pohl

Manihot carthagenensis (Jacq.) Müll. Arg.

Manihot chlorosticta Standl. \& Goldman

Manihot oaxacana D.J. Rogers \& Appan

Omphalea diandra L.

Pleradenophora bilocularis (S. Watson) Esser \& A.L. Melo

Ricinus communis L.

Sapium lateriflorum Hemsl.

Sebastiania adenophora Pax \& K. Hoffm.

Stillingia linearifolia S. Watson*

Tragia amblyodonta (Müll. Arg.) Pax \& K. Hoffm.

Tragia jonesii Radcl. Sm. \& Govaerts 
Apéndice. Continuación.

Fabaceae

Abrus precatorius L.

Acacia californica subsp. pringlei (Rose) L. Rico

Acacia glauca (L.) Moench

Acacia pacensis Rudd \& A.M. Carter

Acaciella goldmanii Britton \& Rose

Acmispon argophyllus (A. Gray) Brouillet

Acmispon distichus (Greene) Brouillet

Acmispon glaber (Vogel) Brouillet

Acmispon niveus (S. Watson) Brouillet

Acmispon prostratus (Nutt.) Brouillet

Acmispon strigosus (Nutt.) Brouillet

Aeschynomene americana $\mathrm{L}$.

Aeschynomene nivea Brandegee

Aeschynomene vigil Brandegee

Agati grandiflora (L.) Desv.

Alysicarpus vaginalis (L.) DC.

Andira galeottiana Standl.

Apoplanesia paniculata C. Presl

Astragalus anemophilus Greene

Astragalus fastidius (Kellogg) M.E. Jones

Astragalus harbisonii Barneby

Astragalus hornii A. Gray

Astragalus insularis Kellogg

Astragalus magdalenae Greene*

Astragalus prorifer M.E. Jones

Astragalus trichopodus (Nutt.) A. Gray

Bauhinia divaricata $\mathrm{L}$.

Bauhinia subrotundifolia Cav.

Bauhinia ungulata L.

Brongniartia peninsularis Rose

Caesalpinia bonduc (L.) Roxb.*

Caesalpinia mexicana A. Gray

Caesalpinia mollis (Kunth) Spreng.

Caesalpinia palmeri S. Watson

Caesalpinia pannosa Brandegee

Caesalpinia placida Brandegee

Caesalpinia pulcherrima (L.) Sw.

Caesalpinia vesicaria L.

Calliandra californica Benth.

Calliandra eriophylla Benth.

Calliandra formosa (Kunth) Benth.

Calopogonium mucunoides Desv.

Canavalia acuminata Rose

Canavalia brasiliensis Mart. ex Benth.

Canavalia ensiformis (L.) DC.
Canavalia rosea (Sw.) DC.*

Canavalia villosa Benth.

Centrosema angustifolium (Kunth) Benth.*

Centrosema plumieri (Turpin ex Pers.) Benth.

Centrosema schottii (Millsp.) K. Schum.

Centrosema virginianum (L.) Benth.

Chamaecrista chamaecristoides (Collad.) Greene*

Chamaecrista diphylla (L.) Greene

Chamaecrista flexuosa (L.) Greene

Chamaecrista glandulosa (L.) Greene

Chamaecrista hispidula (Vahl) H.S. Irwin \& Barneby*

Chamaecrista nictitans (L.) Moench

Chamaecrista rufa (M. Martens \& Galeotti) Britton \& Rose

Chloroleucon mangense (Jacq.) Britton \& Rose

Clitoria ternatea $\mathrm{L}$.

Cojoba arborea (L.) Britton \& Rose

Cologania broussonetii (Balb.) DC.

Coulteria platyloba (S. Watson) N. Zamora

Coursetia caribaea (Jacq.) Lavin

Crotalaria incana L.

Crotalaria pumila Ortega*

Crotalaria purdiana $\mathrm{H}$. Senn

Crotalaria retusa $\mathrm{L}$.

Crotalaria sagittalis L.

Dalbergia brownei (Jacq.) Schinz

Dalbergia ecastaphyllum (L.) Taub.

Dalea bicolor Humb. \& Bonpl. ex Willd.

Dalea brachystachys A. Gray

Dalea carthagenensis (Jacq.) J.F. Macbr.

Dalea cliffortiana Willd.

Dalea mollis Benth.

Dalea mollissima (Rydb.) Munz

Dalea purpusii Brandegee

Dalea tinctoria Brandegee

Delonix regia (Bojer ex Hook.) Raf.

Desmanthus fruticosus Rose

Desmanthus virgatus (L.) Willd.

Desmodium barbatum (L.) Benth.

Desmodium frutescens Schindl.

Desmodium glabrum (Mill.) DC.

Desmodium grahamii A. Gray

Desmodium griffithianum Benth.

Desmodium procumbens (Mill.) Hitchc.

Desmodium purpusii Brandegee

Desmodium scorpiurus (Sw.) Poir.

Desmodium tortuosum (Sw.) DC. 
Apéndice. Continuación.

Diphysa carthagenensis Jacq.

Diphysa yucatanensis A.M. Hanan \& M. Sousa

Ebenopsis ebano (Berland.) Barneby \& J.W. Grimes

Entada polystachya (L.) DC.

Errazurizia megacarpa (S. Watson) I.M. Johnst.

Erythrina americana Mill.

Erythrina berteroana Urb.

Erythrina herbacea L.

Galactia brachystachys Benth.

Galactia jussiaeana Kunth

Galactia striata (Jacq.) Urb.

Gliricidia sepium (Jacq.) Kunth ex Walp.

Haematoxylum campechianum $\mathrm{L}$.

Havardia platyloba (Bertero ex DC.) Britton \& Rose

Hoffmannseggia intricata Brandegee

Hoffmannseggia microphylla Torr.

Indigofera fruticosa Rose

Indigofera hirsuta L.

Indigofera microcarpa Desv.

Indigofera miniata Ortega

Indigofera suffruticosa Mill.

Indigofera trita L. f.*

Inga pinetorum Pittier

Inga punctata Willd.

Inga vera Willd.

Leucaena lanceolata S. Watson

Leucaena leucocephala (Lam.) de Wit

Lonchocarpus guatemalensis Benth.

Lonchocarpus hondurensis Benth.

Lonchocarpus luteomaculatus Pittier

Lonchocarpus rugosus Benth.

Lonchocarpus sericeus (Poir.) Kunth ex DC.

Lonchocarpus yucatanensis Pittier

Lotus nuttallianus Greene

Lotus scoparius (Torr. \& A. Gray) Ottley

Lupinus arizonicus (S. Watson) S. Watson

Lupinus pallidus Brandegee

Lupinus sparsiflorus Benth.

Lupinus succulentus Douglas ex K. Koch

Lupinus truncatus Nutt. ex Hook. \& Arn.

Lysiloma candidum Brandegee

Lysiloma divaricatum (Jacq.) J.F. Macbr.

Lysiloma latisiliquum (L.) Benth.

Macroptilium atropurpureum (Moc. \& Sessé ex DC.) Urb.

Macroptilium gracile (Poepp. ex Benth.) Urb.

Marina brevis León de la Luz*
Marina divaricata (Benth.) Barneby

Marina maritima (Brandegee) Barneby*

Marina oculata (Rydb.) Barneby

Marina parryi (Torr. \& A. Gray) Barneby

Marina vetula (Brandegee) Barneby

Mariosousa centralis (Britton \& Rose) Seigler \& Ebinger

Mariosousa dolichostachya (S.F. Blake) Seigler \& Ebinger

Medicago polymorpha $\mathrm{L}$.

Melilotus indicus (L.) All.

Mimosa antioquensis Killip ex Rudd

Mimosa bahamensis Benth.

Mimosa candollei R. Grether

Mimosa diplotricha C. Wright ex Sauvalle

Mimosa distachya Cav.

Mimosa occidentalis Britton \& Rose

Mimosa pigra L.

Mimosa pudica L.

Mimosa quadrivalvis L.

Mimosa strigillosa Torr. \& A. Gray

Mimosa tricephala Schltdl. \& Cham.

Mucuna sloanei Fawc. \& Rendle

Neptunia plena (L.) Benth.

Neptunia pubescens Benth.

Olneya tesota A. Gray

Parkinsonia aculeata L.

Parkinsonia microphylla Torr.

Phaseolus acutifolius A. Gray

Phaseolus filiformis Benth.

Phaseolus lunatus L.

Phaseolus micranthus Hook. \& Arn.

Piscidia grandifolia (Donn. Sm.) I.M. Johnst.

Piscidia piscipula (L.) Sarg.

Pithecellobium dulce (Roxb.) Benth.

Pithecellobium graciliflorum S.F. Blake

Pithecellobium keyense Britton*

Pithecellobium lanceolatum (Humb. \& Bonpl. ex Willd.) Benth.

Pithecellobium platylobum (Bertero ex DC.) Urb.

Pithecellobium scopulinum (Brandegee) Standl.

Pithecellobium seleri Harms

Pithecellobium unguis-cati (L.) Benth.

Platymiscium pinnatum (Jacq.) Dugand

Prosopidastrum mexicanum (Dressler) Burkart

Prosopis articulata S. Watson

Prosopis glandulosa Torr.

Prosopis juliflora (Sw.) DC.

Prosopis reptans Benth. 
Apéndice. Continuación.

Psorothamnus emoryi (A. Gray) Rydb.

Pterocarpus amphymenium DC.

Rhynchosia americana (Mill.) Metz

Rhynchosia minima (L.) DC.

Samanea saman (Jacq.) Merr.

Senna atomaria (L.) H.S. Irwin \& Barneby

Senna bicapsularis (L.) Roxb.

Senna confinis (Greene) H.S. Irwin \& Barneby

Senna covesii (A. Gray) H.S. Irwin \& Barneby

Senna fruticosa (Mill.) H.S. Irwin \& Barneby

Senna obtusifolia (L.) H.S. Irwin \& Barneby

Senna occidentalis (L.) Link

Senna pallida (Vahl) H.S. Irwin \& Barneby

Senna papillosa (Britton \& Rose) H.S. Irwin \& Barneby

Senna polyphylla (Jacq.) H.S. Irwin \& Barneby

Senna purpusii (Brandegee) H.S. Irwin \& Barneby

Senna racemosa (Mill.) H.S. Irwin \& Barneby

Senna skinneri (Benth.) H.S. Irwin \& Barneby

Senna uniflora (Mill.) H.S. Irwin \& Barneby

Sesbania herbacea (Mill.) McVaugh

Sophora tomentosa L.*

Stylosanthes calcicola Small

Stylosanthes humilis Kunth

Stylosanthes viscosa (L.) Sw.*

Tephrosia cana Brandegee

Tephrosia cinerea (L.) Pers.*

Tephrosia palmeri S. Watson

Tephrosia vicioides Schltdl.

Vachellia campechiana (Mill.) Seigler \& Ebinger

Vachellia collinsii (Saff.) Seigler \& Ebinger

Vachellia constricta (Benth.) Seigler \& Ebinger

Vachellia cornigera (L.) Seigler \& Ebinger

Vachellia farnesiana (L.) Wight \& Arn.

Vachellia macracantha (Humb. \& Bonpl. ex Willd.) Seigler \& Ebinger

Vachellia pennatula (Schltdl. \& Cham.) Seigler \& Ebinger

Vachellia sphaerocephala (Cham. \& Schltdl.) Seigler \& Ebinger

Vigna adenantha (G. Mey.) Maréchal, Mascherpa \& Stainier

Vigna elegans (Piper) Maréchal, Mascherpa \& Stainier

Vigna linearis (Kunth) Maréchal, Mascherpa \& Stainier

Vigna luteola (Jacq.) Benth.

Fagaceae

Quercus oleoides Schltdl. \& Cham.

Fouquieriaceae

Fouquieria burragei Rose
Fouquieria columnaris (Kellogg) Kellogg ex Curran

Fouquieria diguetii (Tiegh.) I.M. Johnst.

Fouquieria splendens Engelm.

Frankeniaceae

Frankenia palmeri $\mathrm{S}$. Watson

Frankenia salina (Molina) I.M. Johnst.

Gentianaceae

Eustoma exaltatum (L.) Salisb. ex G. Don

Leiphaimos parasitica Schltdl. \& Cham.

Sabatia arenicola Greenm.

Geraniaceae

Erodium cicutarium (L.) L'Her. ex Aiton

Gesneriaceae

Smithiantha cinnibarina (Linden) Kuntze

Goodeniaceae

Scaevola plumieri (L.) Vahl*

Hypericaceae

Hypericum pratense Schltdl. \& Cham.

Koeberliniaceae

Koeberlinia spinosa Zucc.

Krameriaceae

Krameria erecta Willd.

Krameria grayi Rose \& J.H. Painter

Krameria paucifolia (Rose) Rose

Lamiaceae

Aegiphila deppeana Steud.

Callicarpa acuminata Kunth

Clerodendrum aculeatum (L.) Schltdl.*

Clerodendrum ligustrinum (Jacq.) R. Br.

Clerodendrum thomsoniae Balf.

Condea albida Kunth Harley \& J.F.B. Pastore

Condea emoryi (Torr.) Harley \& J.F.B. Pastore

Condea laniflora (Benth.) Harley \& J.F.B. Pastore

Condea tephrodes (A. Gray) Harley \& J.F.B. Pastore

Condea verticillata (Jacq.) Harley \& J.F.B. Pastore

Hyptis atrorubens Poit.

Lepechinia hastata (A. Gray) Epling 
Apéndice. Continuación.

Marrubium vulgare L.

Mentha aquatica $\mathrm{L}$.

Salvia coccinea Buc'hoz ex Etl.

Salvia misella Kunth

Salvia munzii Epling

Salvia occidentalis Sw.

Salvia platycheila A. Gray

Salvia similis Brandegee

Teucrium cubense Jacq.

Teucrium glandulosum Kellogg

Vitex gaumeri Greenm.

Vitex mollis Kunth

Linaceae

Linum orizabae Planch.

Linum schiedeanum Schltdl. \& Cham.

Loasaceae

Eucnide cordata Kellogg

Eucnide rupestris (Baill.) H.J. Thomps. \& W.R. Ernst

Mentzelia adhaerens Benth.

Mentzelia aspera $\mathrm{L}$.

Mentzelia multiflora (Nutt.) A. Gray*

Petalonyx thurberi A. Gray

Loganiaceae

Spigelia anthelmia L.

Loranthaceae

Psittacanthus calyculatus (DC.) G. Don

Psittacanthus schiedeanus (Schltdl. \& Cham.) G. Don

Psittacanthus sonorae (S. Watson) Kuijt

Struthanthus cassythoides Millsp. ex Standl.*

Struthanthus crassipes (Oliv.) Eichler

Struthanthus interruptus (Kunth) G. Don

Struthanthus palmeri Kuijt

Struthanthus quercicola (Schltdl. \& Cham.) Blume

Lythraceae

Ammannia auriculata Willd.

Ammannia coccinea Rottb.

Cuphea decandra Aiton

Cuphea gaumeri Koehne

Ginoria nudiflora (Hemsl.) Koehne

Lawsonia inermis L.

Lythrum acinifolium Sessé \& Moc. ex Koehne

Lythrum gracile Benth.
Malpighiaceae

Bunchosia biocellata Schltdl.

Bunchosia lindeniana A. Juss.

Bunchosia palmeri S. Watson

Bunchosia swartziana Griseb.

Byrsonima bucidifolia Standl.

Byrsonima crassifolia (L.) Kunth

Callaeum macropterum (Moc. \& Sessé ex DC.) D.M. Johnson

Cottsia californica (Benth.) W.R. Anderson \& C. Davis

Cottsia gracilis (A. Gray) W.R. Anderson \& C. Davis

Galphimia angustifolia Benth.

Galphimia arenicola C.E. Anderson

Galphimia tuberculata (Rose) Nied.

Heteropterys laurifolia (L.) A. Juss.

Malpighia diversifolia Brandegee

Malpighia glabra L.

Malpighia ovata Rose

Psychopterys dipholiphylla (Small) W.R. Anderson \& S. Corso

Stigmaphyllon bannisterioides (L.) C.E. Anderson

Stigmaphyllon ellipticum (Kunth) A. Juss.

Stigmaphyllon lindenianum A. Juss.

Malvaceae

Abutilon abutiloides (Jacq.) Garcke ex Hochr.

Abutilon berlandieri A. Gray ex S. Watson

Abutilon incanum (Link) Sweet

Abutilon palmeri A. Gray

Abutilon permolle (Willd.) Sweet

Anoda cristata (L.) Schltdl.

Bastardia bivalvis (Cav.) Kunth ex Griseb.

Bastardia viscosa (L.) Kunth

Byttneria aculeata (Jacq.) Jacq.

Ceiba aesculifolia (Kunth) Britten \& Baker f.

Ceiba pentandra (L.) Gaertn.

Cienfuegosia yucatanensis Millsp.

Corchorus aestuans L.

Gossypium davidsonii Kellogg

Gossypium harknessii Brandegee

Gossypium hirsutum L.

Guazuma ulmifolia Lam.

Hampea nutricia Fryxell

Helicteres guazumifolia Kunth

Heliocarpus pallidus Rose

Herissantia crispa (L.) Brizicky

Hermannia palmeri Rose

Hibiscus costatus A. Rich.

Hibiscus denudatus Benth. 
Apéndice. Continuación.

Hibiscus pernambucensis Arruda

Hibiscus rosa-sinensis L.

Horsfordia alata (S. Watson) A. Gray

Horsfordia newberryi (S. Watson) A. Gray

Horsfordia rotundifolia $\mathrm{S}$. Watson

Kosteletzkya depressa (L.) O.J. Blanch., Fryxell \& D.M. Bates

Luehea candida (DC.) Mart.

Malachra alceifolia Jacq.

Malacothamnus fasciculatus (Nutt. ex Torr. \& A. Gray) Greene

Malva parviflora $\mathrm{L}$.

Malvastrum americanum (L.) Torr.

Malvastrum bicuspidatum (S. Watson) Rose

Malvastrum corchorifolium (Desr.) Britton ex Small

Malvastrum coromandelianum (L.) Garcke

Malvaviscus arboreus Cav.

Malvella lepidota (A. Gray) Fryxell

Malvella leprosa (Ortega) Krapov.

Melochia pyramidata $\mathrm{L}$.

Melochia tomentosa L.

Melochia villosa (Mill.) Fawc. \& Rendle

Pachira aquatica Aubl.

Pavonia schiedeana Steud.

Pseudabutilon umbellatum (L.) Fryxell

Pseudobombax ellipticum (Kunth) Dugand

Sida abutilifolia Mill.

Sida acuta Burm. f.

Sida ciliaris L.

Sida cordifolia L.

Sida glabra Mill.

Sida linifolia Juss. ex Cav.

Sida rhombifolia $\mathrm{L}$.

Sida xanti A. Gray

Sphaeralcea ambigua A. Gray

Sphaeralcea coulteri (S. Watson) A. Gray

Sphaeralcea emoryi Torr. ex A. Gray

Sphaeralcea fulva Greene

Sphaeralcea orcuttii Rose

Thespesia populnea (L.) Sol. ex Correa

Trichospermum galeottii (Turcz.) Kosterm.

Triumfetta semitriloba Jacq.

Waltheria americana $\mathrm{L}$.

Wissadula periplocifolia (L.) C. Presl ex Thwaites

Marcgraviaceae

Souroubea loczyi (V.A. Richt.) de Roon
Martyniaceae

Martynia annua L.

Proboscidea altheifolia (Benth.) Decne.

Melastomataceae

Clidemia hirta (L.) D. Don

Conostegia xalapensis (Bonpl.) D. Don ex DC.

Leandra mexicana (Naudin) Cogn.

Miconia argentea (Sw.) DC.

Meliaceae

Cedrela odorata L.

Guarea glabra Vahl

Melia azedarach L.

Trichilia glabra L.

Trichilia hirta L.

Trichilia martiana C. DC.

Trichilia minutiflora Standl.

Trichilia trifolia L. subsp. trifolia

Menispermaceae

Cissampelos owariensis P. Beauv. ex DC.

Cocculus diversifolius DC.

Hyperbaena ilicifolia Standl.

Hyperbaena jalcomulcensis E. Pérez \& Cast.-Campos

Hyperbaena mexicana Miers

Menyanthaceae

Nymphoides indica (L.) Kuntze

Molluginaceae

Glinus radiatus (Ruiz \& Pav.) Rohrb.

Mollugo cerviana (L.) Ser.

Mollugo verticillata $\mathrm{L}$.

Montiaceae

Cistanthe maritima (Nutt.) Carolin ex Hershk.

Moraceae

Brosimum alicastrum Sw.

Castilla elastica Sessé

Dorstenia contrajerva L.

Ficus americana Aubl.

Ficus apollinaris Dugand

Ficus aurea Nutt.

Ficus cotinifolia Kunth 
Apéndice. Continuación.

Ficus crassinervia Desf. ex Willd.

Ficus crocata (Miq.) Miq.

Ficus insipida Willd.

Ficus maxima Mill.

Ficus obtusifolia Kunth

Ficus pertusa L. f.

Ficus petiolaris Kunth

Ficus radula Humb. \& Bonpl. ex Willd.

Ficus velutina Humb. \& Bonpl. ex Willd.

Ficus yoponensis Desv.

Maclura tinctoria (L.) D. Don ex Steud.

Trophis mexicana (Liebm.) Bureau

Trophis racemosa (L.) Urb.

Muntingiaceae

Muntingia calabura L.

Myricaceae

Morella cerifera (L.) Small

Myrtaceae

Calyptranthes karwinskyana O. Berg

Calyptranthes pallens Griseb.

Calyptranthes schiedeana O. Berg

Eugenia acapulcensis Steud.

Eugenia axillaris (Sw.) Willd.

Eugenia capuli (Schltdl. \& Cham.) Hook. \& Arn.

Eugenia karwinskyana O. Berg

Eugenia mexicana Steud.

Eugenia oerstediana O. Berg

Myrcianthes fragrans (Sw.) McVaugh

Psidium guajava L.

Nyctaginaceae

Abronia carterae Ferris

Abronia gracilis Benth.

Abronia maritima Nutt. ex S. Watson*

Abronia umbellata Lam.

Abronia villosa S. Watson*

Allionia incarnata $\mathrm{L}$.

Boerhavia diffusa $\mathrm{L}$.

Boerhavia intermedia M.E. Jones

Boerhavia maculata Standl.

Boerhavia spicata Choisy

Boerhavia triquetra $\mathrm{S}$. Watson

Boerhavia xanti S. Watson
Commicarpus scandens (L.) Standl.

Cryptocarpus pyriformis Kunth

Guapira costaricana (Standl.) Woodson

Mirabilis jalapa L.

Mirabilis laevis (Benth.) Curran

Neea psychotrioides Donn. Sm.

Neea stenophylla Standl.

Okenia hypogaea Schltdl. \& Cham.*

Pisonia aculeata L.

Salpianthus arenarius Bonpl.

Salpianthus macrodontus Standl.

Ochnaceae

Ouratea crassinervia Engl.

Ouratea nitida (Sw.) Engl.

Oleaceae

Forestiera macrocarpa Brandegee

Forestiera rhamnifolia Griseb.

Forestiera shrevei Standl.

Onagraceae

Camissonia angelorum (S. Watson) P.H. Raven

Camissonia californica (Nutt. ex Torr. \& A. Gray) P.H. Raven

Camissonia cardiophylla (Torr.) P.H. Raven

Camissonia cheiranthifolia (Hornem. ex Spreng.) Raim.

Camissonia claviformis (Torr. \& Frém.) P.H. Raven

Camissonia crassifolia (Greene) P.H. Raven

Camissonia lewisii P.H. Raven

Camissonia sceptrostigma (Brandegee) P.H. Raven

Ludwigia octovalvis (Jacq.) P.H. Raven

Oenothera arizonica (Munz) W.L. Wagner

Oenothera deltoides Torr. \& Frém.*

Oenothera drummondii Hook.*

Oenothera pubescens Willd. ex Spreng.

Oenothera wigginsii W.E. Klein*

Xylonagra arborea (Kellogg) Donn. Sm. \& Rose

Opiliaceae

Agonandra obtusifolia Standl.

Orobanchaceae

Agalinis fasciculata (Elliott) Raf.

Agalinis heterophylla (Nutt.) Small

Agalinis linifolia (Nutt.) Britton

Buchnera longifolia Kunth 
Apéndice. Continuación.

Chloropyron maritimum (Nutt. ex Benth.) A. Heller

Dicranostegia orcuttiana (A. Gray) Pennell

Orobanche parishii (Jeps.) Heckard

Oxalidaceae

Oxalis frutescens $\mathrm{L}$.

Papaveraceae

Argemone gracilenta Greene

Argemone ochroleuca Sweet

Eschscholzia californica Cham.

Eschscholzia minutiflora S. Watson

Eschscholzia palmeri Rose

Eschscholzia parishii Greene

Passifloraceae

Passiflora arida (Mast. \& Rose) Killip

Passiflora biflora Lam.

Passiflora ciliata Aiton

Passiflora coriacea Juss.

Passiflora foetida $\mathrm{L}$.

Passiflora fruticosa Killip

Passiflora holosericea L.

Passiflora mexicana Juss.

Passiflora microstipula L.E. Gilbert \& J.M. MacDougal

Passiflora palmeri Rose

Passiflora pavonis Mast.

Passiflora serratifolia L.

Passiflora suberosa L.

Passiflora sublanceolata (Killip) J.M. MacDougal

Passiflora subpeltata Ortega

Passiflora viridiflora Cav.

Turnera diffusa Willd.

Turnera ulmifolia L.

Pentaphylacaceae

Ternstroemia tepezapote Schltdl. \& Cham.

Petiveriaceae

Petiveria alliacea $\mathrm{L}$.

Rivina humilis L.

Trichostigma octandrum (L.) H. Walter

Phrymaceae

Diplacus puniceus Nutt.

Mimulus aurantiacus Curtis
Phyllanthaceae

Andrachne microphylla (Lam.) Baill.

Astrocasia tremula (Griseb.) G.L. Webster

Phyllanthus caroliniensis Walter

Phyllanthus elsiae Urb.

Phyllanthus niruri L.

Phyllanthus nobilis (L. f.) Müll. Arg.

Picramniaceae

Picramnia antidesma subsp. fessonia (DC.) W.W. Thomas

Plantaginaceae

Antirrhinum coulterianum Benth. ex A. DC.

Antirrhinum cyathiferum Benth.

Antirrhinum nuttallianum Benth. ex A. DC.

Antirrhinum watsonii Vasey \& Rose

Collinsia heterophylla Buist ex R. Graham

Conobea polystachya Minod

Gambelia juncea (Benth.) D.A. Sutton

Mecardonia procumbens (Mill.) Small

Nuttallanthus canadensis (L.) D.A. Sutton

Nuttallanthus texanus (Scheele) D.A. Sutton

Plantago major L.

Plantago ovata Forssk.

Russelia coccinea (L.) Wettst.

Russelia equisetiformis Schltdl. \& Cham.

Russelia sarmentosa Jacq.

Russelia tenuis Lundell

Sairocarpus watsonii (Vasey \& Rose) D.A. Sutton

Schistophragma mexicanum Benth. ex D. Dietr.

Scoparia dulcis L.

Stemodia maritima L.*

Stemodia tomentosa G. Don

Plumbaginaceae

Limonium californicum (Boiss.) A. Heller

Limonium carolinianum (Walter) Britton

Limonium sinuatum (L.) Mill.

Plumbago zeylanica L.

Polemoniaceae

Acanthogilia gloriosa (Brandegee) A.G. Day \& Moran

Bryantiella palmeri (S. Watson) J.M. Porter

Gilia palmeri $\mathrm{S}$. Watson

Langloisia setosissima (Torr. \& A. Gray) Greene

Linanthus jonesii (A. Gray) Greene

Linanthus pygmaeus (Brand) J.T. Howell 
Apéndice. Continuación.

Polygalaceae

Polygala alba Nutt.

Securidaca diversifolia (L.) S.F. Blake

Polygonaceae

Antigonon flavescens S. Watson

Antigonon leptopus Hook. \& Arn.

Chorizanthe procumbens Nutt.

Coccoloba acapulcensis Standl.

Coccoloba barbadensis Jacq.

Coccoloba cozumelensis Hemsl.

Coccoloba humboldtii Meisn.

Coccoloba liebmannii Lindau

Coccoloba ortizii R.A. Howard

Coccoloba swartzii Meisn.

Coccoloba uvifera (L.) L.*

Coccoloba venosa $\mathrm{L}$.

Eriogonum fasciculatum Benth.

Eriogonum galioides I.M. Johnst.

Eriogonum grande Greene

Eriogonum inflatum Torr. \& Frém.

Eriogonum moranii Reveal

Eriogonum pondii Greene

Eriogonum preclarum Reveal

Eriogonum repens Reveal

Eriogonum scalare S. Watson

Eriogonum thomasii Torr.

Eriogonum wrightii Torr. ex Benth.

Gymnopodium floribundum Rolfe

Harfordia macroptera (Benth.) Greene \& Parry

Lastarriaea coriacea (Goodman) Hoover

Nemacaulis denudata Nutt.*

Persicaria ferruginea (Wedd.) Soják

Podopterus cordifolius Rose \& Standl.

Podopterus mexicanus Bonpl.

Polygonum argyrocoleon Steud. ex Kunze

Polygonum longiocreatum Bartlett

Pterostegia drymarioides Fisch. \& C.A. Mey.

Rumex conglomeratus Murray

Rumex crispus L.

Rumex maritimus $\mathrm{L}$.

Rumex pulcher L.

Ruprechtia pallida Standl.

Portulacaceae

Portulaca amilis Speg.
Portulaca californica D. Legrand

Portulaca halimoides L.

Portulaca oleracea L.

Portulaca suffrutescens Engelm.

Portulaca umbraticola Kunth

Primulaceae

Anagallis arvensis $\mathrm{L}$.

Ardisia compressa Kunth

Ardisia escallonioides Schltdl. \& Cham.

Ardisia paschalis Donn. Sm.

Ardisia purpurea Reinw.

Ardisia revoluta Kunth

Bonellia flammea (Millsp. ex Mez) B. Ståhl \& Källersjö

Bonellia macrocarpa (Cav.) B. Ståhl \& Källersjö

Jacquinia armillaris Jacq.

Jacquinia aurantiaca W.T. Aiton

Lysimachia ovalis (Ruiz \& Pav.) U. Manns \& Anderb.

Parathesis lenticellata Lundell

Parathesis melanosticta (Schltdl.) Hemsl.

Samolus ebracteatus Kunth

Samolus parviflorus Raf.

Ranunculaceae

Clematis dioica $\mathrm{L}$.

\section{Resedaceae}

Forchhammeria pallida Liebm.

Forchhammeria watsonii Rose

Oligomeris linifolia (Vahl) J.F. Macbr.

Rhamnaceae

Colubrina arborescens (Mill.) Sarg.

Colubrina elliptica (Sw.) Brizicky \& W.L. Stern

Colubrina glabra S. Watson

Colubrina viridis (M.E. Jones) M.C. Johnst.

Condalia globosa I.M. Johnst.

Gouania lupuloides Urb.

Karwinskia humboldtiana (Schult.) Zucc.

Sageretia elegans (Kunth) Brongn.

Ziziphus amole (Sessé \& Moç.) M.C. Johnst.

Ziziphus obtusifolia (Hook. ex Torr. \& A. Gray) A. Gray

Rosaceae

Prunus brachybotrya Zucc. 
Apéndice. Continuación.

Rubiaceae

Alibertia edulis (Rich.) A. Rich. ex DC.

Asemnantha pubescens Hook. f.

Borreria capitata (Ruiz \& Pav.) DC.

Borreria spinosa Cham. \& Schltdl. ex DC.

Borreria tenuior L.

Calycophyllum candidissimum (Vahl) DC.

Chiococca alba (L.) Hitchc.

Chiococca coriacea M. Martens \& Galeotti

Coccocypselum hirsutum Bartl. ex DC.

Crusea longiflora (Willd. ex Roem. \& Schult.) W.R. Anderson

Diodia teres Walter

Erithalis fruticosa L.*

Ernodea littoralis Sw.*

Eumachia microdon (DC.) Delprete \& J.H. Kirkbr.

Exostema caribaeum (Jacq.) Schult.

Faramea occidentalis (L.) A. Rich.

Guettarda elliptica Sw.

Hamelia calycosa Donn. Sm.

Hamelia patens Jacq.

Hedyotis lancifolia Schumach.

Hexasepalum angustifolium Bartl. ex DC.

Hintonia latiflora (DC.) Bullock

Hintonia octomera (Hemsl.) Bullock

Machaonia lindeniana Baill.

Mitracarpus hirtus (L.) DC.

Mitracarpus linearis Benth.

Mitracarpus schizangius DC.

Morinda citrifolia L.

Morinda royoc $\mathrm{L}$.

Oldenlandia corymbosa $\mathrm{L}$.

Palicourea gardenioides (Scheidw.) Hemsl.

Palicourea tetragona (Donn. Sm.) C.M. Taylor \& Lorence

Psychotria calophylla Standl.

Psychotria costiveniaGriseb.

Psychotria erythrocarpa Schltdl.

Psychotria horizontalis Sw.

Psychotria nervosa Sw.

Psychotria oerstediana Standl.

Rachicallis americana (Jacq.) Hitchc.

Randia aculeata $\mathrm{L}$.

Randia armata (Sw.) DC.

Randia capitata DC.

Randia laetevirens Standl.

Randia malacocarpa Standl.

Randia monantha Benth.
Randia obcordata S. Watson

Randia retroflexa Lorence \& M. Nee

Randia tetracantha (Cav.) DC.

Randia thurberi $\mathrm{S}$. Watson

Randia truncata Greenm. \& C.H. Thomps.

Randia xalapensis M. Martens \& Galeotti

Richardia scabra L.

Solenandra mexicana (A. Gray) Borhidi

Spermacoce confusa Rendle

Spermacoce laevis Lam.

Spermacoce remota Lam.

Spermacoce verticillata L.

Stenotis arenaria (Rose) Terrell

Stenotis asperuloides (Benth.) Terrell

Stenotis brevipes (Rose) Terrell

Stenotis mucronata (Benth.) Terrell*

Strumpfia maritima Jacq.

Rutaceae

Amyris balsamifera $\mathrm{L}$.

Esenbeckia berlandieri Baill.

Esenbeckia flava Brandegee

Esenbeckia hartmanii Robinson \& Fernald

Zanthoxylum caribaeum Lam.

Salicaceae

Casearia guevarana Cast.-Campos \& E. Medina

Casearia guianensis (Aubl.) Urb.

Casearia nitida (L.) Jacq.

Casearia obovata Schltdl.

Casearia sylvestris Sw.

Pleuranthodendron lindenii (Turcz.) Sleumer

Xylosma flexuosa (Kunth) Hemsl.

Santalaceae

Phoradendron brachystachyum (DC.) Oliv.

Phoradendron costaricense (Urb.) J.F. Morales

Phoradendron mucronatum (DC.) Krug \& Urb.

Phoradendron nervosum Oliv.

Phoradendron quadrangulare (Kunth) Griseb.

Phoradendron villosum (Nutt.) Nutt. ex Engelm.

Phoradendron wattii Krug \& Urb.*

Sapindaceae

Aesculus parryi A. Gray

Cardiospermum grandiflorum Sw. 
Apéndice. Continuación.

Cardiospermum halicacabum $\mathrm{L}$.

Cardiospermum tortuosum Benth.

Cupania dentata DC.

Cupania macrophylla Mart.

Cupania schippii Standl.

Dodonaea viscosa Jacq.

Exothea paniculata (Juss.) Radlk.

Matayba clavelligera Radlk.

Melicoccus bijugatus Jacq.

Paullinia costaricensis Radlk.

Paullinia fuscescens Kunth

Paullinia pinnata $\mathrm{L}$.

Paullinia tomentosa Jacq.

Sapindus saponaria $\mathrm{L}$.

Serjania adiantoides Radlk.

Serjania atrolineata C. Wright

Serjania macrocarpa Standl. \& Steyerm.

Serjania palmeri $\mathrm{S}$. Watson

Serjania racemosa Schumach.

Serjania triquetra Radlk.

Thouinidium decandrum (Bonpl.) Radlk.

Sapotaceae

Chrysophyllum mexicanum Brandegee ex Standl.

Manilkara zapota (L.) P. Royen

Pouteria campechiana (Kunth) Baehni*

Pouteria hypoglauca (Standl.) Baehni

Sideroxylon americanum (Mill.) T.D. Penn.

Sideroxylon capiri (A. DC.) Pittier

Sideroxylon celastrinum (Kunth) T.D. Penn.

Sideroxylon foetidissimum Jacq.

Sideroxylon obtusifolium (Humb. ex Roem. \& Schult.) T.D. Penn.

Schoepfiaceae

Schoepfia californica Brandegee

Schoepfia schreberi J.F. Gmel.

Scrophulariaceae

Capraria biflora L.

Capraria frutescens (Mill.) Britten

Myoporum laetum $\mathrm{G}$. Forst.

Simaroubaceae

Castela peninsularis Rose

Simmondsiaceae

Simmondsia chinensis (Link) C.K. Schneid.
Solanaceae

Capsicum annuum $\mathrm{L}$.

Cestrum alternifolium (Jacq.) O.E. Schulz

Cestrum dumetorum Schltdl.

Cestrum nocturnum $\mathrm{L}$.

Datura discolor Bernh.

Datura inoxia Mill.

Datura lanosa Barclay ex Bye

Datura stramonium L.

Juanulloa mexicana (Schltdl.) Miers

Lycianthes armentalis J.L. Gentry

Lycianthes lenta (Cav.) Bitter

Lycium andersonii A. Gray

Lycium berlandieri Dunal

Lycium brevipes Benth.

Lycium californicum Nutt. ex A. Gray

Lycium carolinianum Walter*

Lycium exsertum A. Gray

Lycium fremontii A. Gray

Lycium megacarpum Wiggins

Lycium parishii A. Gray

Lycium richii A. Gray

Lycopersicon esculentum Mill.

Nicandra physalodes (L.) Gaertn.

Nicotiana clevelandii A. Gray

Nicotiana glauca Graham

Nicotiana obtusifolia M. Martens \& Galeotti

Physalis acutifolia (Miers) Sandwith

Physalis cinerascens (Dunal) Hitchc.

Physalis crassifolia Benth.

Physalis glabra Benth.

Physalis maxima Mill.

Physalis mayana Standl.

Physalis minuta Griggs

Physalis pubescens L.

Physalis solanacea (Schltdl.) Axelius

Solanum americanum Mill.

Solanum candidum Lindl.

Solanum diphyllum $\mathrm{L}$.

Solanum donianum Walp.

Solanum elaeagnifolium Cav.

Solanum erianthum D. Don

Solanum hindsianum Benth.

Solanum lycopersicum L.

Solanum nudum Dunal

Solanum palmeri Vasey \& Rose

Solanum rudepannum Dunal 
Apéndice. Continuación.

Solanum tampicense Dunal

Solanum tridynamum Dunal

Solanum umbellatum Mill.

Stegnospermataceae

Stegnosperma cubense A. Rich.

Stegnosperma halimifolium Benth.

Surianaceae

Suriana maritima L.

Talinaceae

Talinum paniculatum (Jacq.) Gaertn.

Talinum triangulare (Jacq.) Willd.

Tamaricaceae

Tamarix ramosissima Ledeb.

Tetrachondraceae

Polypremum procumbens L.

Thymelaeaceae

Daphnopsis americana (Mill.) J.R. Johnst.

Urticaceae

Cecropia peltata L.

Parietaria debilis G. Forst.

Verbenaceae

Aloysia sonorensis Moldenke

Burroughsia fastigiata (Brandegee) Moldenke

Citharexylum ellipticum D. Don

Citharexylum hexangulare Greenm.

Duranta repens L.

Lantana camara L.

Lantana hirta Graham

Lantana involucrata L.

Lippia formosa Brandegee

Lippia nodiflora (L.) Michx.

Lippia palmeri $\mathrm{S}$. Watson

Lippia stoechadifolia (L.) Kunth

Petrea volubilis L.

Priva lappulacea (L.) Pers.

Stachytarpheta jamaicensis (L.) Vahl

Stachytarpheta miniacea Moldenke

Tamonea curassavica (L.) Pers.

Verbena halei Small
Violaceae

Hybanthus calceolaria (L.) Oken

Hybanthus fruticulosus (Benth.) I.M. Johnst.

Orthion oblanceolatum Lundell

Vitaceae

Ampelopsis denudata Planch.

Cissus alata Jacq.

Cissus microcarpa Vahl

Cissus sicyoides L.

Vitis bourgaeana Planch.

Vitis cinerea (Engelm.) Millardet

Vitis tiliifolia Humb. \& Bonpl. ex Schult.

Vochysiaceae

Vochysia guatemalensis Donn. Sm.

Ximeniaceae

Ximenia americana $\mathrm{L}$.

Ximenia pubescens Standl.

Zygophyllaceae

Fagonia barclayana (Benth.) Rydb.

Fagonia californica Benth.

Fagonia laevis Standl.

Fagonia pachyacantha Rydb.

Fagonia palmeri Vasey \& Rose

Fagonia villosa D.M. Porter

Guaiacum coulteri A. Gray

Guaiacum sanctum L.

Guaiacum unijugum Brandegee

Kallstroemia californica (S. Watson) Vail

Kallstroemia curta Rydb.

Kallstroemia maxima (L.) Hook. \& Arn.

Kallstroemia peninsularis D.M. Porter

Kallstroemia pubescens (G. Don) Dandy

Larrea tridentata (DC.) Coville

Tribulus terrestris L.

Viscainoa geniculata (Kellogg) Greene

Zygophyllum pterocarpum Bunge 